Mon. Not. R. Astron. Soc. 000, 000-000 (0000) Printed 3 November $2018 \quad$ (MN LATEX style file v2.2)

\title{
How Many CVs are Crossing the Period Gap? A Test for the Disruption of Magnetic Braking
}

\author{
P. J. Davis,${ }^{1}$ U. Kolb, ${ }^{1}$ B. Willems ${ }^{2}$ B. T. Gänsicke, ${ }^{3}$ \\ ${ }^{1}$ The Open University, Department of Physics and Astronomy, Walton Hall, Milton Keynes MK 7 6AA \\ ${ }^{2}$ Northwestern University, Department of Physics and Astronomy, 2131 Tech Drive, Evanston, IL 60208, USA \\ ${ }^{3}$ The University of Warwick, Department of Physics and Astronomy, Coventry CV4 7AL
}

3 November 2018

\begin{abstract}
We apply population synthesis techniques to calculate the present day number of two types of white dwarf-main sequence star (WDMS) binaries within the cataclysmic variable 2 to 3 hour period gap. The first are post-common envelope binaries with secondary stars that have masses $0.17 \leq M_{2} / \mathrm{M}_{\odot} \leq 0.36(\mathrm{gPCEBs})$, such that they will commence mass transfer within the period gap. The second type are systems that were CVs at some point in their past, but detached once they evolved down in orbital period to $\approx 3 \mathrm{~h}$ as a consequence of disrupted magnetic braking, and are crossing the period gap via gravitational radiation (dCVs). Full population synthesis calculations are performed where we assume either constant, global values of the common envelope ejection efficiency, $\alpha_{\mathrm{CE}}$, or consider $\alpha_{\mathrm{CE}}$ as a function of secondary mass. Several forms of magnetic braking are also considered. We predict an excess of dCVs over gPCEBs within the period gap of $\sim 4$ to $\sim 13$ assuming $\alpha_{\mathrm{CE}}=0.1-0.6$, and an initial mass ratio distribution of the form $n(q)=1$. This excess is revealed as a prominent peak at the location of the period gap in the orbital period distribution of the combined gPCEB and $\mathrm{dCV}$ population. We suggest that if such a feature is observed in the orbital period distribution of an observed sample of short orbital period WDMS binaries, this would strongly corroborate the disruption of magnetic braking.
\end{abstract}

Key words: binaries: close - methods: statistical - novae, cataclysmic variables stars: evolution

\section{INTRODUCTION}

The standard paradigm of cataclysmic variable (CV) evolution assumes that orbital angular momentum (AM) is lost via both gravitational radiation and magnetic braking at orbital periods $P_{\text {orb }} / \mathrm{h}>3$, and purely by gravitational radiation for $P_{\text {orb }} / \mathrm{h} \lesssim 3$ (e.g. Warner 1995; King 1988). However, the AM loss rate associated with magnetic braking is ill-constrained, and it is unclear if magnetic braking ceases to be a sink of AM for $P_{\text {orb }} / \mathrm{h} \lesssim 3$ Kolb 2002).

Extrapolating the work of Skumanich (1972) who investigated the spin down rates of G-type stars by a magnetically coupled stellar wind, Verbunt \& Zwaan (1981) obtained the magnetic braking AM loss rate of the form $\dot{J}_{\mathrm{MB}} \propto \Omega^{3}$, where $\Omega$ is the spin frequency of the donor star.

As a consequence of the AM losses and the mass loss they drive, the donor star is unable to maintain thermal equilibrium. This causes donors with masses $\lesssim 0.8 \mathrm{M}_{\odot}$ to exceed their equilibrium radius Stehle. Ritter \& Kolb 1996). Mass loss will eventually cause the donor star to become fully convective at a mass $M_{2}=M_{2, \text { conv }}$ and when $P_{\text {orb }} / \mathrm{h} \approx 3$. At this point it is suggested that either the mag- netic activity of the donor star discontinuously decreases, or the topology of the magnetic field changes (Taam \& Spruit 1989). This is based on the result that the interface between the radiative core and the convective envelope play a key role in the generation of the magnetic field (McDermott \& Taam 1989).

When magnetic braking ceases at $M_{2}=M_{2, \text { conv }}$ the donor shrinks within its Roche lobe, mass transfer is shut off and the secondary re-attains thermal equilibrium. The system is no longer observed as a CV. Subsequent orbital evolution of the $\mathrm{CV}$ is driven by gravitational radiation only. Mass transfer resumes again when the secondary star makes contact with its Roche lobe once more at $P_{\text {orb }} / \mathrm{h} \approx 2$.

This disrupted magnetic model was conceived to explain the lack of observed CVs in the range $2 \lesssim P_{\text {orb }} / \mathrm{h} \lesssim 3$ (Rappaport, Verbunt \& Joss 1983; Spruit \& Ritter 1983), as shown in Figure 1

However, despite the success of this model in reproducing the correct width and position of the period gap (e.g. Kolb, King \& Ritter 1998), there is no independent observational support for disrupted magnetic braking. Ob- 


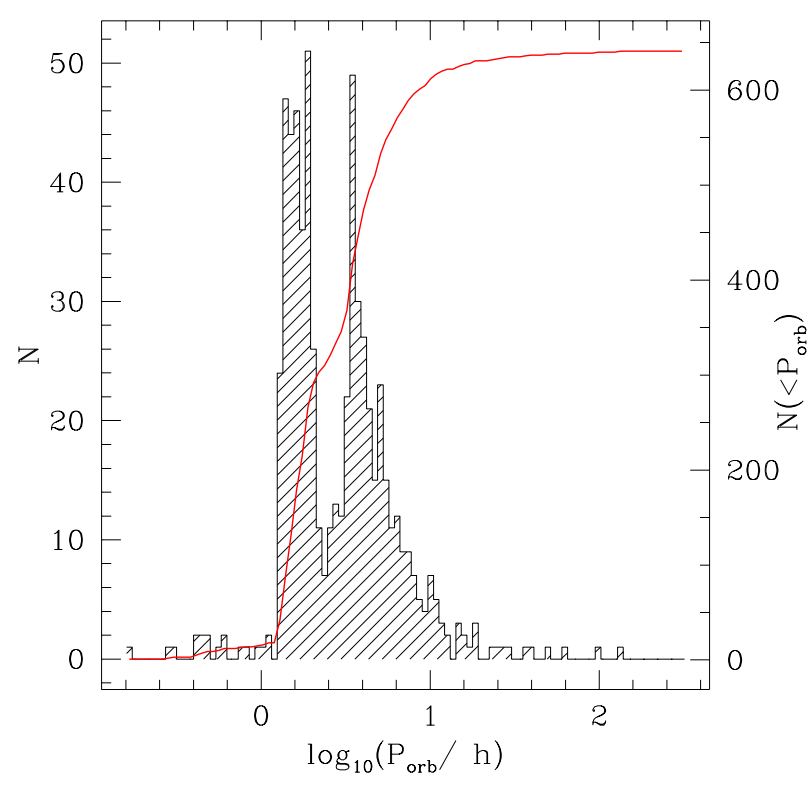

Figure 1. Orbital period distribution of CVs from Ritter \& Kolb (2003), catalogue edition 7.7 (2006). The axis on the left indicates the number of systems per bin. The solid, red curve is the cumulative distribution with numbers, $N<P_{\text {orb }}$, indicated on the right axis.

servations of young, open clusters (Sills, Pinsonneault \& Terndrup 2000) show that the spin down rate of rapidly rotating single stars is much less than predicted by the Verbunt \& Zwaan (1981) relation. Also, no discontinuous decline in magnetic activity for the fully convective stars is detected (Jones, Fischer \& Stauffer 1996). It is likely that for the high rotational speeds of $\gtrsim 100 \mathrm{~km} \mathrm{~s}^{-1}$ of the $\mathrm{CV}$ donor stars, the AM loss rate, $\dot{J}_{\mathrm{MB}}$, due to magnetic braking will saturate (for a review see e.g. Collier-Cameron 2002). Sills et al. (2000) suggest the form $\dot{J}_{\mathrm{MB}} \propto \Omega$ in the saturated regime. Within this scheme the AM loss rate in CVs would be a factor of 100 less than that for the standard magnetic braking model, and the donor star would not be sufficiently driven out of thermal equilibrium and no period gap would arise (Andronov, Pinsonneault \& Sills 2003).

Such theoretical and observational problems with the standard model of CV evolution aside, it is the only one thus far that predicts a period gap of the correct width with edges at the correct orbital periods 1 . Two recent pieces of evidence in support for the disrupted magnetic braking model lies in the mass-radius relation of donor stars in CVs (Smith \& Dhillon 1998; Patterson et al. 2005; Knigge 2006) and in the effective temperatures of the accretion-heated white dwarfs in CVs (Townsley \& Bildsten 2003). In the former, the donor mass-radius relation for $P_{\text {orb }} / \mathrm{h} \gtrsim 3$ lies systematically above that for isolated main sequence stars. This is consistent with the donor star being driven out of

1 Willems et al. (2005) and Willems et al. (2007) studied CV populations evolving under the influence of circumbinary disks and found a period gap feature, but had to appeal to additional mechanisms in the pre- $\mathrm{CV}$ evolution to account for the lower edge of the gap. thermal equilibrium. Furthermore, a discontinuous decrease in the donor radii between $2 \lesssim P_{\text {orb }} / \mathrm{h} \lesssim 3$ has been detected. In the latter piece of evidence, the effective temperatures of white dwarfs are higher above the period gap than below, and are overall consistent with the expected accretion heating for mass transfer rates predicted by the disrupted magnetic braking model.

Politano \& Weiler (2006) calculated the secondary star mass distribution of present day PCEBs for a range of AM loss prescriptions, including disrupted magnetic braking. They found that the number of PCEBs per secondary mass interval decreases by 38 per cent once magnetic braking begins to drive their evolution for secondaries with radiative cores. They suggest that this decrease in the number of PCEBs with secondaries between $\sim 0.25$ and $0.5 \mathrm{M}_{\odot}$ provides an observable test for the disruption of magnetic braking.

In this paper we suggest an alternative, even more direct, test for the disruption of magnetic braking by investigating a more explicit consequence: the existence of a population of WDMS binaries that were CVs in the past but became detached at $P_{\text {orb }} / \mathrm{h} \approx 3$ and are currently crossing the period gap. We call these dCVs.

Using population synthesis techniques we calculate the present day population of these dCVs, as well as WDMS binaries that have emerged from a common envelope (CE) phase. We call these systems post-CE binaries (PCEBs). In particular, we only consider PCEBs which will begin mass transfer within the period gap. For our stellar models and choice of gap boundaries, such systems have secondary masses in the range $0.17 \leq M_{2} / \mathrm{M}_{\odot} \leq 0.36$. This sub-set of PCEBs we henceforth designate as gPCEB systems. Note that gPCEBs have $P_{\text {orb }} / \mathrm{h} \geq 2$ but can also have $P_{\text {orb }} / \mathrm{h}>3$.

As we will show below orbital the period distribution of the combined population of dCVs and gPCEBs reveals a prominent peak within the period gap due to an excess of $\mathrm{dCVs}$ over gPCEBs here. We calculate the ratio $\mathrm{dCV}$ :gPCEB within the period gap, which indicates the height of this peak, for a range of models concerning the values of the CE ejection efficiency, $\alpha_{\mathrm{CE}}$, the initial distribution of secondary mass, and the AM loss rate due to magnetic braking. The detection of such a feature in the orbital period distribution of an observed sample of WDMS binaries with orbital periods of a few hours would provide a simple test for the disruption of magnetic braking.

The Sloan Digital Sky Survey (SDSS) is currently creating the opportunity for large-scale observational studies of WDMS binaries, having identified already more than 1000 systems (Silvestri et al. 2007; Schreiber, Nebot GomezMoran \& Schwope 2007). Attempts to establish the subsample of PCEBs among those systems and measuring their orbital periods as well as their stellar properties is underway (Rebassa-Mansergas et al. 2007, Schreiber et al. 2008). We are optimistic that a quantitative test of the calculations carried out in this paper, and hence of the validity of the disrupted magnetic braking hypothesis, will be feasible in the near future.

The structure of the paper is as follows. In Section 2 we describe the computational method, in Section 3 we present our simulation results, which are discussed in Section 4. We conclude our investigation in Section 5 . 


\section{COMPUTATIONAL METHOD}

The computational method we apply consists of two main steps; the first is to calculate the zero-age populations of the dCVs (i.e. CVs that have just detached at the upper edge of the period gap) and PCEBs (i.e. WDMS binaries that have just emerged from a CE phase) using the population synthesis code BiSEPS (Willems \& Kolb 2002; Willems \& Kolb 2004). These populations are unweighted i.e. no formation probabilities are yet calculated for the systems. The code employs the single star evolution (SSE) formulae described in Hurley, Pols \& Tout (2000) and a binary system evolution scheme based on that described in Hurley, Pols \& Tout (2002). The second step uses a different code introduced in Willems et al. (2005), which calculates the present day populations of these systems using a library of evolutionary tracks, here produced by BiSEPS itself. The procedure is described in more detail in the following sections.

\subsection{Initial Binary Population}

BiSEPS evolves a large number of binary systems, which initially consist of two zero-age main sequence stellar components. The stars are assumed to have a population I chemical composition and the orbits are circular at all times. The initial primary and secondary masses are in the range 0.1 to $20 \mathrm{M}_{\odot}$, while the initial orbital periods range from 0.1 to $100000 \mathrm{~d}$. There is one representative binary configuration per grid cell within a three-dimensional grid consisting of 60 logarithmically spaced points in primary and secondary mass and 300 logarithmically spaced points in orbital period. There are $\sim 5.4 \times 10^{6}$ binaries that are evolved for a maximum evolution time of 10 Gyr. For symmetry reasons only systems with $M_{1}>M_{2}$ are evolved.

We calculate the four dimensional probability density functions $\chi\left(t, M_{\mathrm{WD}}, M_{2}, P_{\mathrm{orb}}\right)$ of all zero-age $\mathrm{dCVs}$ and $\phi\left(t, M_{\mathrm{WD}}, M_{2}, P_{\mathrm{orb}}\right)$ for all zero-age PCEBs using the initial distribution functions quoted below. Here $t$ is the time since the birth of the Galaxy, $M_{\mathrm{WD}}$ is the mass of the white dwarf, $M_{2}$ is the mass of the secondary star and $P_{\text {orb }}$ is the orbital period. The number of zero-age main sequence primaries, $\mathrm{d} N$, that form with masses in the range $\mathrm{d} M_{1}$ is given by $\mathrm{d} N=f\left(M_{1}\right) \mathrm{d} M_{1}$, where the probability density function, $f\left(M_{1}\right)$, is given by

$$
f\left(M_{1}\right)= \begin{cases}0 & M_{1} / \mathrm{M}_{\odot}<0.1 \\ 0.29056 M_{1}^{-1.3} & 0.1 \leq M_{1} / \mathrm{M}_{\odot}<0.5, \\ 0.15571 M_{1}^{-2.2} & 0.5 \leq M_{1} / \mathrm{M}_{\odot}<1.0 \\ 0.15571 M_{1}^{-2.7} & 1.0 \leq M_{1} / \mathrm{M}_{\odot},\end{cases}
$$

(Kroupa, Tout \& Gilmore 1993). The probability that a zeroage main sequence secondary star forms with a mass $M_{2}$ can be determined from the initial mass ratio distribution (IMRD) given by

$n(q)= \begin{cases}\mu q^{\nu} & 0<q \leq 1 \\ 0 & q>1\end{cases}$

where $q=M_{2} / M_{1}, \nu$ is a constant, and $\mu$ is a normalisation factor such that $\int n(q) \mathrm{d} q=1(\mu=\nu+1$ for $\nu>-1)$. We also consider the case where the secondary mass is determined from an IMF according to equation (1) where $M_{1}$ is replaced with $M_{2}$. We use $n(q)=1$, i.e. $\nu=0$, as our reference model. Finally, the probability that a binary forms with an initial orbital separation $a$ is determined by (Iben \& Tutukov 1984; Hurley et al. 2002)

$$
h(a)= \begin{cases}0 & a / \mathrm{R}_{\odot}<3 \text { or } a / \mathrm{R}_{\odot}>10^{6}, \\ 0.078636 a^{-1} & 3 \leq a / \mathrm{R}_{\odot} \leq 10^{6} .\end{cases}
$$

We further assume that all stars in the Galaxy are formed in binaries and that the Galaxy has an age of 10 Gyr. The star formation rate, $S$, is calculated by assuming that one binary per year is formed with $M_{1}>0.8 \mathrm{M}_{\odot}$. Combining this with the Galactic volume of $5 \times 10^{11} \mathrm{pc}^{3}$ gives an average local birthrate of white dwarfs of $2 \times 10^{-12}$ $\mathrm{pc}^{-3} \mathrm{yr}^{-1}$, consistent with observations (Weidemann 1990). The lower limit of $0.8 \mathrm{M}_{\odot}$ is the smallest mass the primary star can have if it is to evolve into a white dwarf within the lifetime of the Galaxy. We therefore have

$S \int_{0.8}^{\infty} f\left(M_{1}\right) \mathrm{d} M_{1}=1 \mathrm{yr}^{-1}$.

From (1) and (4) we obtain $S=7.6 \mathrm{yr}^{-1}$. We assume that this is constant throughout the lifetime of the Galaxy.

We then employ a population calculator to convert $\chi\left(t, M_{\mathrm{WD}}, M_{2}, P_{\mathrm{orb}}\right)$ and $\phi\left(t, M_{\mathrm{WD}}, M_{2}, P_{\mathrm{orb}}\right)$ into distributions over $M_{\mathrm{WD}}, M_{2}$ and $P_{\text {orb }}$ describing the present day population of dCVs and gPCEBs. The secular evolution of each system in the zero-age populations is therefore followed using BiSEPS, and its contribution to the population, determined by the above distribution function and star formation rate, is accounted for at each stage of its evolution.

\subsection{Binary Evolution}

The CE phase (Paczyński 1976) is modelled by equating the binding energy of the primary's envelope to the change in the total orbital energy of the binary system. Quantitatively this is

$$
\frac{G M_{\mathrm{e}}\left(M_{\mathrm{e}}+M_{\mathrm{c}}\right)}{\lambda R_{1, \mathrm{~L}}}=\alpha_{\mathrm{CE}}\left[\frac{G M_{\mathrm{c}} M_{2}}{2 a_{\mathrm{f}}}-\frac{G\left(M_{\mathrm{c}}+M_{\mathrm{e}}\right) M_{2}}{2 a_{\mathrm{i}}}\right] \text {, }
$$

where $G$ is the gravitational constant, $M_{\mathrm{c}}$ and $M_{\mathrm{e}}$ are the masses of the primary's core (the proto-white dwarf) and envelope, $R_{1, \mathrm{~L}}$ is the radius of the primary immediately before the onset of the $\mathrm{CE}$ phase, and $M_{2}$ is the mass of the secondary. The orbital separation of the stellar components immediately before and after the $\mathrm{CE}$ phase are $a_{\mathrm{i}}$ and $a_{\mathrm{f}}$ respectively. The constant $\alpha_{\mathrm{CE}}$ is the fraction of the orbital energy used to unbind the envelope from the core of the primary and $\lambda$ is the ratio between the approximate form of the binding energy $G M_{\mathrm{e}}\left(M_{\mathrm{e}}+M_{\mathrm{c}}\right) / R_{1, \mathrm{~L}}$ and the true binding energy. Throughout our investigation we use $\lambda=0.5$, which is consistent with the value used by Willems \& Kolb (2004). We take $\alpha_{\mathrm{CE}}=1.0$ as our reference model 'A'.

Once the CE has been ejected from the system, then if the binary's stellar components have avoided a collision, what remains is a PCEB system. The subsequent evolution of the system is driven by orbital AM losses; a combination of magnetic braking and gravitational radiation if the secondary mass is larger than the mass of a fully convective, isolated main sequence star, $M_{\mathrm{MS} \text {,conv }}$, and purely by gravitational radiation otherwise.

The system will become semi-detached once the orbital separation has decreased sufficiently so that the secondary 
Table 1. Model assumptions for $\alpha_{\mathrm{CE}}$ and magnetic braking.

\begin{tabular}{|c|c|c|}
\hline Model & $\alpha_{\mathrm{CE}}$ & Magnetic braking law \\
\hline $\mathrm{hA}$ & 1.0 & eqn. (8) \\
\hline hCE01 & 0.1 & eqn. (8) \\
\hline hCE06 & 0.6 & eqn. (8) \\
\hline hCE3 & 3.0 & eqn. (8) \\
\hline hCE5 & 5.0 & eqn. (8) \\
\hline hPWR05 & eqn.(6),$p=0.5$ & eqn. (8) \\
\hline hPWR1 & 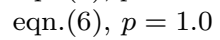 & eqn. (8) \\
\hline hPWR2 & eqn.([6), $p=2.0$ & eqn. (8) \\
\hline $\mathrm{rvj} 2 \mathrm{~A}$ & 1.0 & eqn. (7), $\gamma=2$ \\
\hline $\mathrm{rvj} 4 \mathrm{~A}$ & 1.0 & eqn. (7), $\gamma=4$ \\
\hline
\end{tabular}

star is brought into contact with its Roche lobe. If the mass ratio $q \lesssim 1.3$ then mass transfer driven by AM losses will ensue. If, however, $q \gtrsim 1.3$ then the secondary will undergo thermal timescale mass transfer (TTMT). Depending on the initial mass ratio and evolutionary state, some of these systems may reappear as AM-driven CVs. It is thought that as many as 40 per cent Kolb \& Willems 2005) of the present population of CVs may have formed in this way. The detection of $\mathrm{CNO}$ abundance anomalies supports this hypothesis, placing a lower limit of 10 per cent on the fraction of post-TTMT systems among the observed population of CVs (Gänsicke et al. 2003). Determination of the parameters at which the TTMT phase reverts to the standard AMdriven phase would require a comprehensive treatment of the TTMT phase, and would depend on the fate of the transferred material (Schenker 2001). We therefore only consider the contribution of purely $\mathrm{AM}$ driven $\mathrm{CVs}$ in the present investigation.

\subsubsection{The Ejection Efficiency Parameter, $\alpha_{\mathrm{CE}}$}

In our calculations, we consider the impact of changing the common envelope ejection efficiency $\alpha_{\mathrm{CE}}$. In addition to constant, global values of $\alpha_{\mathrm{CE}}$ we follow Politano \& Weiler (2007) and consider the possibility that $\alpha_{\mathrm{CE}}$ could be a function of the secondary mass. We adopt

$\alpha_{\mathrm{CE}}=\left(\frac{M_{2}}{\mathrm{M}_{\odot}}\right)^{p}$

where $p$ is a free parameter. Figure 2 shows how $\alpha_{\mathrm{CE}}$ varies with $M_{2}$ for $p=0.5,1.0$ and 2.0. The dark-grey shading is the range of secondary masses in gPCEBs considered here, while the light-grey shading is the range of secondary masses found for dCV progenitors. Table 1 summarises our population synthesis models for varying values of $\alpha_{\mathrm{CE}}$. In our model names, the first portion in lower case denotes the form of magnetic braking used. In Table 1 ' $h$ ' denotes that the Hurley et al. (2002) form of magnetic braking is used according to equation (8). The label ' $\mathrm{CE} x$ ' denotes the value, $x$, of the constant, global value of $\alpha_{\mathrm{CE}}$. For example 'CE01' is $\alpha_{\mathrm{CE}}=0.1$. Finally, the acronym 'PWRy' denotes the use of equation (6) with the value, $y$, of the parameter $p$, for example 'PWR05' is $p=0.5$.

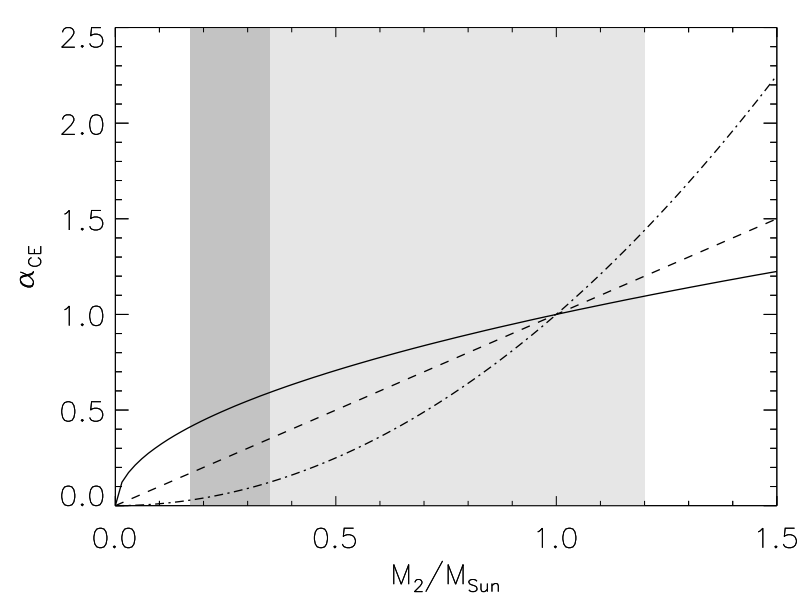

Figure 2. Variation of $\alpha_{\mathrm{CE}}$ with $M_{2}$ according to eqn. (6). Solid: $p=0.5$; dashed: $p=1.0$; dot-dashed: $p=2.0$. Dark-grey shading indicates range of secondary masses in gPCEB systems; light-grey range of secondary masses for $\mathrm{dCV}$ progenitors.

\subsubsection{Magnetic Braking}

We also investigate how various forms of magnetic braking affect our results. Rappaport et al. (1983) give a parameterised form of magnetic braking as

$\dot{J}_{\mathrm{rvj}}=-\eta_{\mathrm{rvj}} 3.8 \times 10^{-30} R_{\odot}^{4} \mathrm{~s} \mathrm{~cm}^{2} M_{2}\left(\frac{R_{2}}{R_{\odot}}\right)^{\gamma} \Omega^{3}$,

where $\Omega$ is the spin frequency of the secondary star and $\gamma$ is a free parameter. If $\gamma=4$ then (7) reduces to the form derived by Verbunt \& Zwaan (1981). The dimensionless constant $\eta_{\mathrm{rvj}}$ is determined so as to give the correct AM loss rate at the upper edge of the period gap, which we determine in Section 2.2.3. The prescription given by Hurley et al. (2002) is given by

$\dot{J}_{\mathrm{h}}=-\eta_{\mathrm{h}} 5.83 \times 10^{-16} \mathrm{M}_{\odot} \mathrm{R}_{\odot}^{-1} \mathrm{yr} \frac{M_{\text {env }}}{M_{2}} R_{2}^{3} \Omega^{3}$,

where $M_{\mathrm{env}}$ is the mass of the secondary star's convective envelope and $R_{2}$ is the donor's radius. Thus, magnetic braking vanishes for a star with no convective envelope. As with equation (7) the value of the constant $\eta_{\mathrm{h}}$ is determined to give the appropriate AM loss rate at the upper edge of the period gap. We use equation (8) for our reference model. Table 1 summarises our models where we use different forms of magnetic braking. The acronym 'rvj2' and 'rvj4' denotes that we use the Rappaport et al. (1983) form of magnetic braking with $\gamma=2$ and 4 respectively.

\subsubsection{The Period Gap}

In this section we outline how we determine the position, and therefore the width, of the period gap. We then ascertain the values of $\eta_{\mathrm{rvj}}$ and $\eta_{\mathrm{h}}$, which will give the AM loss rate at the upper edge of the period gap appropriate for its determined width. Mass loss from the donor will cause it to exceed its equilibrium radius (see Section 2.2 .5 ) by a factor $f_{\text {conv }}$ at the upper edge of the period gap. We calculate this value at the end of this section.

We obtain the position of the gap edges by calculating a moving average of the cumulative distribution curve 
shown in Figure 1. Linear fits were performed on three segments of the curve; within and on either side of the period gap. Specifically, we consider the curve in the range $0.22 \leq \log _{10}\left(P_{\text {orb }} / \mathrm{h}\right) \leq 0.64$. The edges of the period gap are where these fits intersect. We find that the upper and lower edges are located at 3.0 and 2.0 hours respectively. We adopt these values throughout the rest of our investigation.

The magnetic braking laws described in Section 2.2.2 are now calibrated so as to give the correct AM loss rate, and therefore the correct mass transfer rate, at the upper edge of the period gap. Kolb (1996) determined that the mass transfer rate at the upper edge of the period gap is $\approx 10^{-9}$ $\mathrm{M}_{\odot} \mathrm{yr}^{-1}$. For the Rappaport et al. (1983) form of magnetic braking we find that $\eta_{\mathrm{rvj}}=0.28$ for $\gamma=2$. For $\gamma=4$ we find $\eta_{\mathrm{rvj}}=1.5$. For the Hurley et al. (2002) prescription, $\eta_{\mathrm{h}}=0.19$.

We now determine the value of $f_{\text {conv }}$. The Roche lobe radius of the donor star, $R_{2, \mathrm{~L}}$, from Roche geometry

$R_{2, \mathrm{~L}} \approx 0.44\left(\frac{M_{2}}{M_{2}+M_{1}}\right)^{1 / 3} a$,

(Paczyński 1971) and Kepler's law gives

$P_{\text {orb }} \propto\left(\frac{R_{2, \mathrm{~L}}^{3}}{M_{2}}\right)^{1 / 2}$.

Hence the orbital periods of the upper $\left(P_{\mathrm{u}}\right)$ and lower $\left(P_{\ell}\right)$ edges of the period gap relate to the corresponding donor's radii at these locations, $R_{\mathrm{L}, \mathrm{u}}$ and $R_{\mathrm{L}, \ell}$ as

$\frac{P_{\mathrm{u}}}{P_{\ell}}=\left(\frac{R_{\mathrm{L}, \mathrm{u}}}{R_{\mathrm{L}, \ell}}\right)^{3 / 2}$.

There is no term in $M_{2}$ as the mass of the secondary star remains constant across the period gap. Given that $P_{\mathrm{u}} / P_{\ell}=$ $3 / 2$, then $R_{\mathrm{L}, \mathrm{u}} / R_{\mathrm{L}, \ell} \equiv f_{\text {conv }} \approx 1.3$.

\subsubsection{Mass Transfer in CVs}

The instantaneous mass transfer rate, $\dot{M}_{2}$, from the CV donor with radius $R_{2}$ and Roche lobe radius $R_{2, \mathrm{~L}}$ is given by (Ritter 1988)

$-\dot{M}_{2} \approx \dot{M}_{0} \exp \left(\frac{R_{2, \mathrm{~L}}-R_{2}}{H}\right)$,

where $H \approx 10^{-4} R_{2}$ is the photospheric scale height of the donor star and $\dot{M}_{0} \approx 10^{-8} \mathrm{M}_{\odot} \mathrm{yr}^{-1}$. We assume steady state mass transfer, i.e. $\ddot{M}_{2}=0$. From equation (12), this assumption implies that the donor radius and Roche lobe radius move in step

$\frac{\mathrm{d}}{\mathrm{dt}}\left(R_{2, \mathrm{~L}}-R_{2}\right)=0$,

where we have also assumed that $R_{2}-R_{2, \mathrm{~L}} \ll R_{2}$.

We calculate the mass transfer rate for a given binary configuration time step by considering the relative difference between the donor radius and Roche lobe radius $\delta=\left(R_{2}-\right.$ $\left.R_{2, \mathrm{~L}}\right) / R_{2}$ from BiSEPS as a function of trial values of the mass transfer rate. We locate the root of the function $\delta\left(\dot{M}_{2}\right)$ by employing a bisection algorithm, and therefore obtaining the value of $\dot{M}_{2}$. In practice we iterate until we find a value of $\dot{M}_{2}$ where $|\delta| \lesssim 10^{-5}$. This procedure is similar to that used by Kalogera et al. (2003), although our method has been developed independently.
The bisection algorithm encounters numerical problems for those CVs that are close to thermal or dynamical instability, or where the secondary star has evolved significantly from the zero-age main sequence (typically where $\left.M_{2} / M_{\odot} \gtrsim 1.0\right)$. Specifically, the algorithm obtains unphysically high mass transfer rates at the onset of mass transfer. Consequently, the evolutionary tracks of these systems across the period gap are not calculated.

We estimate the present day number of dCVs that these systems would produce for each model by multiplying the mean dCV lifetime with the formation rate of these numerically unstable CVs (we apply equation (25) described in Section 3.1). The mean dCV lifetime, $\left\langle\tau_{\mathrm{dCV}}\right\rangle$, was calculated by evolving a typical $\mathrm{CV}$ with a $0.4 \mathrm{M}_{\odot}$ donor and a $0.6 \mathrm{M}_{\odot}$ white dwarf. These typical values were determined by calculating the probability distribution of all zero-age CVs. We find that $\left\langle\tau_{\mathrm{dCV}}\right\rangle \approx 1053 \mathrm{Myr}$.

The estimated present day number of these dCVs are added onto the present day numbers calculated from the population synthesis. The fraction of the total dCV model population that these numerically unstable CVs contribute are as follows. For the Hurley et al. (2002) prescription of magnetic braking, the contribution is $\lesssim 8.0$ per cent. For the Rappaport et al. (1983) prescription, the contribution is $\lesssim 20.0$ per cent when $\gamma=2$ and $\lesssim 30.0$ per cent when $\gamma=4$.

\subsubsection{The Reaction of the Donor to Mass Loss}

We apply a simple analytical scheme that mimics the reaction of the donor star to mass loss. The expansion of a low-mass donor as a result of mass loss is a well established result from full stellar evolution calculations and analytical considerations (Stehle et al. 1996). We define the radius excess, $f$, as the factor by which the star has exceeded its equilibrium radius, $R_{2 \text {,eq }}$, due to mass loss. We parameterise the evolution of $f$ according to a power law expression

$f \equiv \frac{R_{2, \mathrm{CV}}}{R_{2, \mathrm{eq}}}=A M_{2}^{\beta}$,

where $A$ and $\beta$ are constants.

At the upper edge of the period gap, the donor star exceeds its equilibrium radius by a factor $f=f_{\text {conv }}=1.3$. At the point where mass transfer just commences at $M_{2}=$ $M_{2,0}$, we require that the donor star is in thermal equilibrium i.e. $f=f_{0}=1$. Thus we have

$f_{0}=A M_{2,0}^{\beta}$,

and

$f_{\text {conv }}=A M_{2, \text { conv }}^{\beta}$,

Combining (14), (15) and (16) yields

$f=f_{\text {conv }}\left(\frac{M_{2}}{M_{2, \text { conv }}}\right)^{\beta}$,

where

$\beta=\frac{\ln \left(f_{0} / f_{\text {conv }}\right)}{\ln \left(M_{2,0} / M_{2, \text { conv }}\right)}$.

The value of the donor's radius calculated by BiSEPS is then multiplied by $f$. We assume furthermore that the 


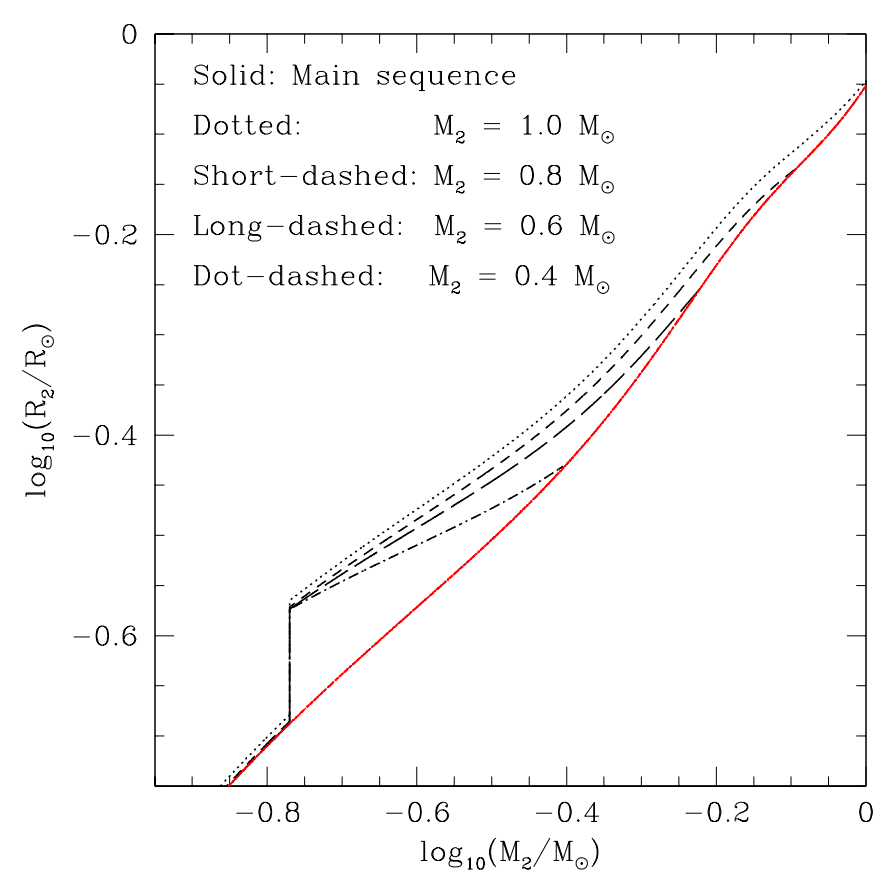

Figure 3. Mass-radius relations for CVs with different initial donor masses.

donor's effective temperature, $T_{\text {eff }}$, is the same as its isolated, main sequence equivalent (Stehle et al. 1996). The luminosity, $L_{2}$, of the donor star is calculated from the StefanBoltzmann Law

$L_{2}=4 \pi{R_{2}}^{2} \sigma T_{\mathrm{eff}}^{4}$.

where $\sigma$ is Stefan-Boltzmann constant. Hence $L_{2}$ will be larger by a factor of $f^{2}$ compared to models without mass loss. The mass-radius relation of several CV evolutionary sequences calculated with this prescription is shown in Figure 3.

In order for the lower edge of the period gap to be in the correct location of $P_{\ell}=2.0 \mathrm{~h}$, we disrupt magnetic braking when $M_{2}=M_{2, \text { conv }}=0.17 \mathrm{M}_{\odot}$.

Based on homology relations, Stehle et al. (1996) derived a first order differential equation that describes how the donor star's radius reacts to mass loss. This scheme shows that CVs with different initial system parameters at the onset of mass transfer quickly converge to a single, uniform evolutionary track, consistent with the well defined position of the period gap.

Our initial scheme to describe the reaction of the donor to mass loss in terms of the mass loss timescale $\tau_{\dot{M}_{2}}$ and its Kelvin-Helmholtz timescale $\tau_{\mathrm{KH}}$, was closely based on that of Stehle et al. (1996), according to

$f=\kappa \frac{\tau_{\mathrm{KH}}}{\tau_{\dot{M}_{2}}}+1$,

where the value of the constant $\kappa$ can be determined by stipulating that $f=f_{\text {conv }}=1.3$ at the upper edge of the period gap. However, we encountered numerical instabilities due to feedback between the donor radius and the mass transfer rate. The scheme we use instead does not reproduce convergence of $\mathrm{CV}$ evolution onto a single track, but does ensure that the donors do exceed their equilibrium radius by the same amount by the time they become fully convective. As such, we ensure that we reproduce the observed width of the period gap with a well defined location. As we are interested in the evolution of systems across the period gap, rather than the detailed $\mathrm{CV}$ evolution above the gap, we believe the prescription we adopt is satisfactory.

Following King \& Kolb (1995) once the donor star detaches from its Roche lobe it re-attains its equilibrium radius on the timescale

$\tau=\left(1-\frac{1}{f_{\text {conv }}}\right) \frac{D}{\zeta_{\text {eff }}-\zeta_{\text {ad }}} \tau_{J}$,

where $\zeta_{\text {eff }}=\mathrm{d} \ln R_{2} / \mathrm{d} \ln M_{2}$ is the effective mass-radius index, $\zeta_{\text {ad }}$ is the adiabatic mass-radius exponent, $\tau_{J}$ is the angular momentum loss rate timescale and the stability denominator $D \approx 1$ is given by equation (16) in King \& Kolb (1995). These constants are evaluated just before the CV becomes detached at the upper edge of the period gap. Typically, $\tau \approx 10^{7} \mathrm{yr}$. In the detached phase the radius excess follows the relation

$f(t)=1-\left(1-f_{\text {conv }}\right) e^{-t / \tau}$,

where $t$ is the time since the donor detached from its Roche lobe.

\section{RESULTS AND ANALYSIS}

We now present the results of our population synthesis calculations. These are summarised in Table 2 which lists the present day population of dCVs, gPCEBs and the ratio $\mathrm{dCV}$ :gPCEB in the period gap. For completeness we also list the total present day formation rates of all PCEBs, and CVs above and below the period gap. In all our models, we also consider different forms of the IMRD, with $\nu=-0.99,0.0$ or 1.0. We also determine the secondary mass from the same IMF as the primary star. Our reference IMRD is $n(q)=1$.

The excess of dCVs over gPCEBs is illustrated in the top panel of Figure 4] a prominent peak within the period gap in the orbital period distributions of the combined dCV and gPCEB populations. These have been calculated from our reference model hA, for different assumptions on the initial distribution of the secondary mass. The cut-off in the number of systems at 2.0 hours is because the smallest secondary mass considered in both the dCV and gPCEB systems is $0.17 \mathrm{M}_{\odot}$, as such systems become semi-detached at 2.0 hours. The bottom panel of Figure 4 shows the separate orbital period distributions of dCVs (red) and gPCEBs (blue) for our reference model hA, and for $n(q)=1$. The ratio $\mathrm{dCV}$ :gPCEB within the period gap gives an indication of the height of the peak.

\subsection{Varying $\alpha_{\mathrm{CE}}$ : Models hA to hCE5}

We begin by examining how the present day populations of $\mathrm{dCV}$ s and gPCEBs within the period gap are affected by varying the value of the global parameter $\alpha_{\mathrm{CE}}$ for $n(q)=1$ (Figure 5). An understanding of these trends will yield an understanding of the overall trend of dCV:gPCEB with $\alpha_{\mathrm{CE}}$. 

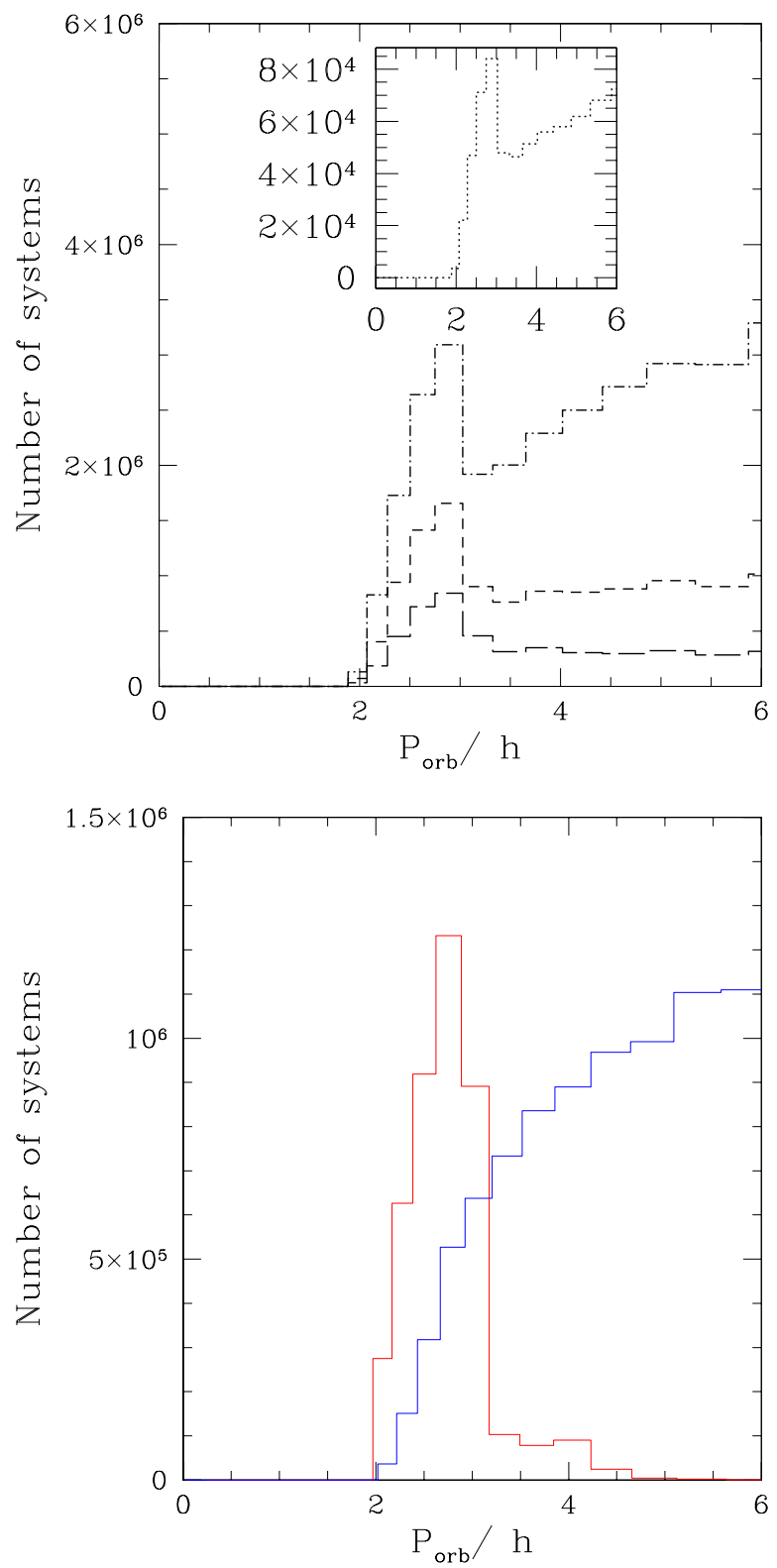

Figure 4. Top panel: orbital period distribution of dCVs and gPCEBs combined for our reference model hA, and for different initial mass ratio distributions. Long-dashed: $n(q) \propto q$; shortdashed: $n(q)=1$ (our reference IMRD); dot-dashed: where $M_{2}$ is picked independently from the same IMF as $M_{1}$ according to equation (1); dotted: $n(q) \propto q^{-0.99}$ (inset). The peak apparent in all the distributions is due to the population of dCVs; the gPCEB orbital period distributions are increasing monotonically with $P_{\text {orb }}$. Bottom panel: orbital period distribution of dCVs (red) and gPCEBs (blue) for our reference model hA, and for $n(q)=1$.

\subsubsection{The gPCEB Population}

The left panel of Figure 5 shows that increasing the value of $\alpha_{\mathrm{CE}}$ from 0.1 to 1.0 increases the present day number of gPCEBs within the period gap (from $1.7 \times 10^{5}$ to $1.0 \times 10^{6}$ for our reference IMRD). This levels off (here to $1.1 \times 10^{6}$ ) for $\alpha_{\mathrm{CE}} \gtrsim 1.0$.
To understand this consider Figure 6, where the dashed box $C D E F$ defined by $0.17 \leq M_{2} / \mathrm{M}_{\odot} \leq 0.36$ and $\log _{10}\left(P_{\ell}\right) \leq P_{\text {orb }} / \mathrm{h} \leq \log _{10}\left(P_{\mathrm{u}}\right)$ shows the location of gPCEBs in the period gap. We designate this region as $\Re$. Not only will $\Re$ consist of gPCEBs that formed there, but also of gPCEBs that evolve into $\Re$ from longer orbital periods. If $t_{\mathrm{u}}=10 \mathrm{Gyr}$ is the maximum time allowed for the gPCEB to reach the upper edge of the period gap and $t_{\mathrm{GR}}$ is the AM loss timescale, $J / \dot{J}$, due to gravitational radiation, then the largest orbital period from which a gPCEB can evolve into the period gap within the lifetime of the Galaxy, $P_{\Re}^{+}$, can be solved from

$t_{\mathrm{u}}=\frac{t_{\mathrm{GR}}\left(P_{\Re}^{+}\right)-t_{\mathrm{GR}}\left(P_{\mathrm{u}}\right)}{8}$,

(e.g. Kolb \& Stehle 1996). The location of $P_{\Re}^{+}$is also shown in Figure 6] Therefore, the total area from which gPCEBs can evolve into $\Re$ within the Galactic lifetime is given by the region $A B C D$.

The whole population of gPCEBs is also bound within an upper limit, $P_{\mathrm{PCEB}}^{+}$(shown in Figure 17), and the Roche lobe-filling limit (RLFL) which is the orbital period where systems become semi-detached. As the value of $\alpha_{\mathrm{CE}}$ is decreased the boundary $P_{\mathrm{PCEB}}^{+}$and the population bound within will shift to shorter orbital periods. This is because, for low ejection efficiencies, more orbital energy is required to eject the envelope from the system. The resulting PCEB (and therefore gPCEB) population will lie at shorter orbital periods.

We find that the flux of gPCEBs from $A B C D$ into $\Re$ is larger than the birthrate of gPCEBs within $\Re$ by a factor of $\sim 7$. If the typical lifetime of a gPCEB within $\Re$ is $\left\langle\tau_{\mathrm{gPCEB}}\right\rangle \approx 380 \mathrm{Myr}$ and the flux of gPCEBs from $A B C D$ into $\Re$ is $F_{\Re}$ then the present day number of gPCEBs within the period gap, $N_{\mathrm{gPCEB}}$, can be approximated as

$N_{\mathrm{gPCEB}} \approx F_{\Re} \times\left\langle\tau_{\mathrm{gPCEB}}\right\rangle$.

Clearly $F_{\Re}$ will depend on the formation rate of gPCEBs in $A B C D$. Figure 7 shows the formation rate of gPCEBs as a function of $\log _{10}\left(P_{\mathrm{orb}} / \mathrm{d}\right)$ for $n(q)=1$, and for $\alpha_{\mathrm{CE}}=0.1$ (red), 1.0 (black) and 5.0 (green). The distribution function is flat-topped and tails off towards longer orbital periods. As $\alpha_{\mathrm{CE}}$ increases and the whole population of gPCEBs shifts to longer orbital periods, the formation rate of the gPCEBs between $\log _{10}\left(P_{\mathrm{u}}\right)$ and $P_{\Re}^{+}$(inset)- and therefore in $A B C D$ changes as a consequence; at its smallest for $\alpha_{\mathrm{CE}}=0.1 \mathrm{but}$ with little change for $\alpha_{\mathrm{CE}} \gtrsim 1.0$.

From equation (24) the trend in $N_{\mathrm{gPCEB}}$ with $\alpha_{\mathrm{CE}}$ will therefore mirror that of the birthrate of gPCEBs in $A B C D$. This is indeed the case as shown in the left panel of Figure 5

\subsubsection{The dCV Population}

As with the present day population of gPCEBs, there is also an increase in the present day number of dCVs associated with an increase in $\alpha_{\mathrm{CE}}$ from 0.1 to 1.0, as shown in the middle panel of Figure 5 For our reference IMRD, the number of dCVs increases from $2.2 \times 10^{6}$ to $3.5 \times 10^{6}$. In contrast to the gPCEB population however, the number of $\mathrm{dCVs}$ decreases with a further increase in the value of $\alpha_{\mathrm{CE}}$.

If the formation rate of $\mathrm{dCVs}$ is $B_{\mathrm{dCV}}$ and their average 
Table 2. Present day population of dCVs, gPCEBs and the ratio dCV:gPCEB in the period gap for various values of $\alpha_{\mathrm{CE}}$ and various forms of magnetic braking. Also quoted is the present day formation rate of all PCEBs, and of CVs above and below the upper edge of the period gap.

\begin{tabular}{|c|c|c|c|c|c|c|}
\hline Model & $\begin{array}{l}\text { Number of } \\
\text { dCVs }\end{array}$ & $\begin{array}{l}\text { Number of } \\
\text { gPCEBs }\end{array}$ & $\begin{array}{l}\text { Formation rate of } \\
\text { PCEBs }\left(\mathrm{yr}^{-1}\right)\end{array}$ & $\begin{array}{l}\text { Formation rate } \\
\text { of } \mathrm{CVs} \geq 3.0 \mathrm{~h} \\
\qquad\left(\mathrm{yr}^{-1}\right)\end{array}$ & $\begin{array}{c}\text { Formation rate } \\
\text { of } \mathrm{CVs}<3.0 \mathrm{~h} \\
\qquad\left(\mathrm{yr}^{-1}\right)\end{array}$ & dCV:gPCEB \\
\hline \multicolumn{7}{|c|}{$n(q) \propto q^{-0.99}, 0<q \leq 1$} \\
\hline $\mathrm{hCE} 01$ & $5.7 \times 10^{4}$ & $8.7 \times 10^{3}$ & $2.1 \times 10^{-4}$ & $6.7 \times 10^{-5}$ & $3.7 \times 10^{-5}$ & 6.6 \\
\hline hCE06 & $1.5 \times 10^{5}$ & $6.1 \times 10^{4}$ & $1.5 \times 10^{-3}$ & $1.8 \times 10^{-4}$ & $2.3 \times 10^{-4}$ & 2.5 \\
\hline $\mathrm{hA}$ & $1.5 \times 10^{5}$ & $7.4 \times 10^{4}$ & $2.0 \times 10^{-3}$ & $1.8 \times 10^{-4}$ & $2.7 \times 10^{-4}$ & 2.0 \\
\hline hCE3 & $9.5 \times 10^{4}$ & $8.2 \times 10^{4}$ & $3.1 \times 10^{-3}$ & $1.1 \times 10^{-4}$ & $2.4 \times 10^{-4}$ & 1.2 \\
\hline hCE5 & $5.9 \times 10^{4}$ & $7.9 \times 10^{4}$ & $3.5 \times 10^{-3}$ & $7.7 \times 10^{-5}$ & $2.0 \times 10^{-4}$ & 0.7 \\
\hline hPWR05 & $1.6 \times 10^{5}$ & $5.2 \times 10^{4}$ & $1.6 \times 10^{-3}$ & $1.8 \times 10^{-4}$ & $1.9 \times 10^{-4}$ & 3.1 \\
\hline hPWR1 & $1.4 \times 10^{5}$ & $2.5 \times 10^{4}$ & $1.3 \times 10^{-3}$ & $1.7 \times 10^{-4}$ & $1.0 \times 10^{-4}$ & 5.6 \\
\hline hPWR2 & $1.0 \times 10^{5}$ & $3.3 \times 10^{3}$ & $1.1 \times 10^{-3}$ & $1.4 \times 10^{-4}$ & $2.2 \times 10^{-5}$ & 30.3 \\
\hline rvj2A & $2.2 \times 10^{5}$ & $7.4 \times 10^{4}$ & $2.0 \times 10^{-3}$ & $2.5 \times 10^{-4}$ & $2.8 \times 10^{-4}$ & 3.0 \\
\hline rvj4A & $2.4 \times 10^{5}$ & $7.4 \times 10^{4}$ & $2.0 \times 10^{-3}$ & $3.2 \times 10^{-4}$ & $2.8 \times 10^{-4}$ & 3.2 \\
\hline \multicolumn{7}{|c|}{$n(q)=1.0,0<q \leq 1($ our reference IMRD) } \\
\hline$\overline{\mathrm{hCE} 01}$ & $2.2 \times 10^{6}$ & $1.7 \times 10^{5}$ & $9.8 \times 10^{-3}$ & $3.1 \times 10^{-3}$ & $7.0 \times 10^{-4}$ & 12.9 \\
\hline hCE06 & $3.8 \times 10^{6}$ & $8.6 \times 10^{5}$ & $5.5 \times 10^{-2}$ & $5.6 \times 10^{-3}$ & $3.3 \times 10^{-3}$ & 4.4 \\
\hline hA & $3.5 \times 10^{6}$ & $1.0 \times 10^{6}$ & $7.2 \times 10^{-2}$ & $5.0 \times 10^{-3}$ & $3.3 \times 10^{-3}$ & 3.5 \\
\hline hCE3 & $2.0 \times 10^{6}$ & $1.1 \times 10^{6}$ & $1.0 \times 10^{-1}$ & $2.7 \times 10^{-3}$ & $2.5 \times 10^{-3}$ & 1.8 \\
\hline hCE5 & $1.0 \times 10^{6}$ & $1.1 \times 10^{6}$ & $1.2 \times 10^{-1}$ & $1.5 \times 10^{-3}$ & $1.9 \times 10^{-3}$ & 0.9 \\
\hline hPWR05 & $4.1 \times 10^{6}$ & $7.7 \times 10^{5}$ & $6.8 \times 10^{-2}$ & $5.2 \times 10^{-3}$ & $2.8 \times 10^{-3}$ & 5.3 \\
\hline hPWR1 & $3.9 \times 10^{6}$ & $4.4 \times 10^{5}$ & $6.5 \times 10^{-2}$ & $5.2 \times 10^{-3}$ & $1.9 \times 10^{-3}$ & 8.9 \\
\hline hPWR2 & $3.1 \times 10^{6}$ & $7.2 \times 10^{4}$ & $6.1 \times 10^{-2}$ & $4.8 \times 10^{-3}$ & $6.8 \times 10^{-4}$ & 43.1 \\
\hline$\overline{r v j 2 A}$ & $5.5 \times 10^{6}$ & $1.0 \times 10^{6}$ & $7.2 \times 10^{-2}$ & $7.6 \times 10^{-3}$ & $3.6 \times 10^{-3}$ & 5.5 \\
\hline rvj4A & $6.0 \times 10^{6}$ & $1.0 \times 10^{6}$ & $7.2 \times 10^{-2}$ & $1.0 \times 10^{-2}$ & $3.9 \times 10^{-3}$ & 6.0 \\
\hline \multicolumn{7}{|c|}{$n(q) \propto q, 0<q \leq 1$} \\
\hline$\overline{\mathrm{hCE} 01}$ & $1.8 \times 10^{6}$ & $6.4 \times 10^{4}$ & $1.1 \times 10^{-2}$ & $3.2 \times 10^{-3}$ & $3.1 \times 10^{-4}$ & 28.1 \\
\hline hCE06 & $2.3 \times 10^{6}$ & $2.7 \times 10^{5}$ & $5.8 \times 10^{-2}$ & $4.2 \times 10^{-3}$ & $1.4 \times 10^{-3}$ & 8.5 \\
\hline $\mathrm{hA}$ & $2.0 \times 10^{6}$ & $3.1 \times 10^{5}$ & $7.5 \times 10^{-2}$ & $3.6 \times 10^{-3}$ & $1.1 \times 10^{-3}$ & 6.5 \\
\hline hCE3 & $9.9 \times 10^{5}$ & $3.6 \times 10^{5}$ & $1.1 \times 10^{-1}$ & $1.7 \times 10^{-3}$ & $6.1 \times 10^{-4}$ & 2.8 \\
\hline hCE5 & $4.1 \times 10^{5}$ & $3.7 \times 10^{5}$ & $1.2 \times 10^{-1}$ & $7.0 \times 10^{-4}$ & $4.5 \times 10^{-4}$ & 1.1 \\
\hline hPWR05 & $2.3 \times 10^{6}$ & $2.5 \times 10^{5}$ & $7.7 \times 10^{-2}$ & $3.7 \times 10^{-3}$ & $1.1 \times 10^{-3}$ & 9.2 \\
\hline hPWR1 & $2.3 \times 10^{6}$ & $1.6 \times 10^{5}$ & $7.8 \times 10^{-2}$ & $3.7 \times 10^{-3}$ & $9.1 \times 10^{-4}$ & 14.4 \\
\hline hPWR2 & $2.0 \times 10^{6}$ & $3.2 \times 10^{4}$ & $7.8 \times 10^{-2}$ & $3.5 \times 10^{-3}$ & $5.3 \times 10^{-4}$ & 62.5 \\
\hline rvj2A & $3.2 \times 10^{6}$ & $3.1 \times 10^{5}$ & $7.5 \times 10^{-2}$ & $5.7 \times 10^{-3}$ & $1.5 \times 10^{-3}$ & 10.3 \\
\hline rvj4A & $3.6 \times 10^{6}$ & $3.1 \times 10^{5}$ & $7.5 \times 10^{-2}$ & $8.9 \times 10^{-3}$ & $1.9 \times 10^{-3}$ & 11.6 \\
\hline \multicolumn{7}{|c|}{$M_{2}$ from IMF according to eqn. (1) } \\
\hline$\overline{\mathrm{hCE} 01}$ & $1.8 \times 10^{6}$ & $4.0 \times 10^{5}$ & $6.5 \times 10^{-3}$ & $1.9 \times 10^{-3}$ & $1.7 \times 10^{-3}$ & 4.5 \\
\hline hCE06 & $4.7 \times 10^{6}$ & $2.9 \times 10^{6}$ & $4.7 \times 10^{-2}$ & $4.8 \times 10^{-3}$ & $1.1 \times 10^{-2}$ & 1.6 \\
\hline hA & $5.0 \times 10^{6}$ & $3.5 \times 10^{6}$ & $6.5 \times 10^{-2}$ & $4.8 \times 10^{-3}$ & $1.3 \times 10^{-2}$ & 1.4 \\
\hline hCE3 & $3.1 \times 10^{6}$ & $3.9 \times 10^{6}$ & $1.0 \times 10^{-1}$ & $3.1 \times 10^{-3}$ & $1.2 \times 10^{-2}$ & 0.8 \\
\hline hCE5 & $1.8 \times 10^{6}$ & $3.7 \times 10^{6}$ & $1.2 \times 10^{-1}$ & $2.1 \times 10^{-3}$ & $1.0 \times 10^{-2}$ & 0.5 \\
\hline hPWR05 & $5.2 \times 10^{6}$ & $2.4 \times 10^{6}$ & $4.4 \times 10^{-2}$ & $4.9 \times 10^{-3}$ & $9.0 \times 10^{-3}$ & 2.2 \\
\hline hPWR1 & $4.7 \times 10^{6}$ & $1.2 \times 10^{6}$ & $2.9 \times 10^{-2}$ & $4.6 \times 10^{-3}$ & $4.3 \times 10^{-3}$ & 3.9 \\
\hline hPWR2 & $3.1 \times 10^{6}$ & $1.4 \times 10^{5}$ & $1.7 \times 10^{-2}$ & $3.6 \times 10^{-3}$ & $8.2 \times 10^{-4}$ & 22.1 \\
\hline rvj2A & $6.2 \times 10^{6}$ & $3.5 \times 10^{6}$ & $6.5 \times 10^{-2}$ & $6.3 \times 10^{-3}$ & $1.4 \times 10^{-2}$ & 1.8 \\
\hline rvj4A & $6.8 \times 10^{6}$ & $3.5 \times 10^{6}$ & $6.5 \times 10^{-2}$ & $7.6 \times 10^{-3}$ & $1.4 \times 10^{-2}$ & 1.9 \\
\hline
\end{tabular}



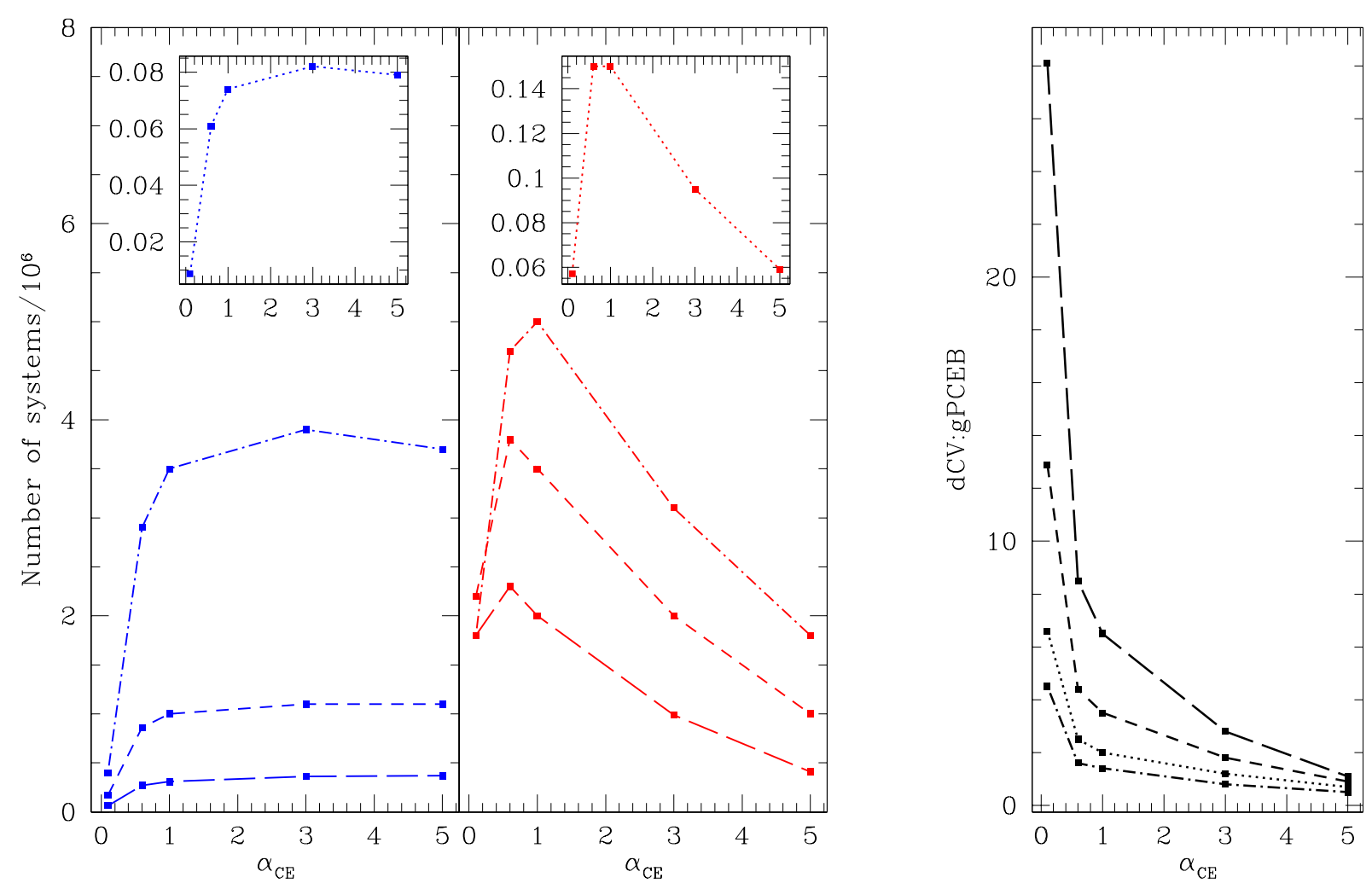

Figure 5. From left to right: the present day population of gPCEBs in the period gap (blue) and dCVs (red) as a function of $\alpha_{\mathrm{CE}}$, and the ratio dCV:gPCEB as a function of $\alpha_{\mathrm{CE}}$. Long-dashed: $n(q) \propto q$; short-dashed: $n(q)=1$ (our reference IMRD); dot-dashed: where $M_{2}$ is picked independently from the same IMF as $M_{1}$ according to equation (1D); dotted: $n(q) \propto q^{-0.99}$ (insets).

lifetime is $\left\langle\tau_{\mathrm{dCV}}\right\rangle$, then the present-day population of dCVs can be approximated by

$N_{\mathrm{dCV}} \approx B_{\mathrm{dCV}} \times\left\langle\tau_{\mathrm{dCV}}\right\rangle$.

If we now assume that the populations above the period gap of pre-CVs (with $M_{2}>M_{\mathrm{MS} \text {,conv }}$ ) and CVs are in a steady state, we then have for their respective formation rates $B_{\text {preCV }}$ and $B_{\mathrm{CV}}$

$B_{\mathrm{PreCV}} \approx B_{\mathrm{CV}} \approx B_{\mathrm{dCV}}$.

The formation rate of $\mathrm{dCVs}$ is therefore linked to that of these pre-CVs above the period gap.

Figure 8 shows the present-day formation rate of preCVs for $n(q)=1$ on the $M_{2}-\log _{10}\left(P_{\text {orb }}\right)$ plane, for $\alpha_{\mathrm{CE}}=0.1$ (top panel), 1.0 (middle panel) and 5.0 (bottom panel), where the colour bar at the top indicates the formation rate in $\mathrm{Myr}^{-1}$ per bin area. The lower boundary in each of the populations is the RLFL, while the upper boundary is given by those PCEBs where the time taken for the primary progenitor to fill its Roche lobe plus the time taken for the secondary to subsequently come into contact with its Roche lobe is 10 Gyr (i.e. the Galactic lifetime). The shape of the upper boundary is a consequence of the AM loss mechanism; gravitational radiation for systems with $M_{2} \leq M_{\mathrm{MS} \text {,conv }}$ or a combination of magnetic braking and gravitational radiation for systems with $M_{2}>M_{\mathrm{MS} \text {,conv }}$.

Inspecting the upper and middle panels of Figure 8 shows that there is an increase in the formation rate of pre-
CVs from $\alpha_{\mathrm{CE}}=0.1$ to $\alpha_{\mathrm{CE}}=1.0$. This is a consequence of the whole population of PCEBs being shifted to longer orbital periods for larger ejection efficiencies, and hence more systems surviving the CE phase. For $\alpha_{\mathrm{CE}}=5.0$ in the lower panel, however, there is an overall decrease in the formation rate of pre-CVs. Progenitor systems with even shorter initial orbital periods than those for models hCE01 or hA can now survive the $\mathrm{CE}$ phase. Such progenitors result in preCVs that formed through a case B CE phase with a naked helium star remnant. These systems lie below the solid line in the bottom panel of Figure 8 while those above the line are those pre-CVs that formed through a case $\mathrm{C}$ CE phase. The latter systems have ended up at much longer orbital periods than for models hCE01 and hA. The solid line represents those pre-CVs whose progenitor primaries just filled their Roche lobes on the base of the AGB. It is the disappearance of case $\mathrm{C}$ remnants and the appearance of the less abundant case $\mathrm{B}$ remnants that causes the decrease in the total pre-CV formation rate.

From equations (25) to (26) this trend in $B_{\text {preCV }}$ should be mirrored in the present day number of $\mathrm{dCVs}$, which is the case as shown in the middle panel of Figure 5

\subsubsection{Overall Trends}

These trends in the populations of gPCEBs and dCVs are the same for all forms of the IMRD function, and where the 


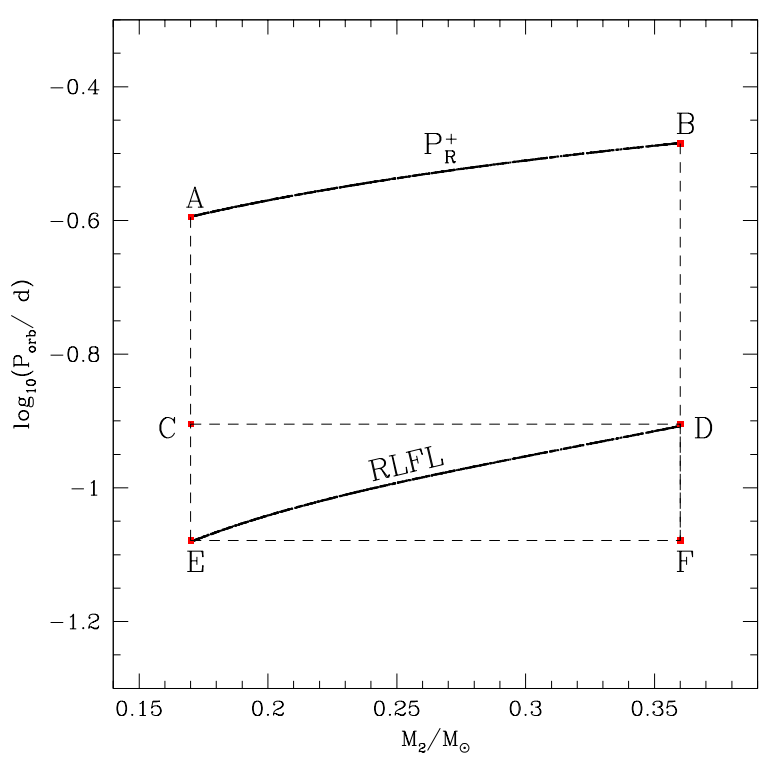

Figure 6. The dashed box, $C D E F$, shows the region $\Re$ defined by $0.17 \leq M_{2} / \mathrm{M}_{\odot} \leq 0.36$ and $\log _{10}\left(P_{\ell}\right) \leq \log _{10}\left(P_{\text {orb }} / \mathrm{d}\right) \leq$ $\log _{10}\left(P_{\mathrm{u}}\right)$. The gPCEBs within $\Re$ will fill their Roche lobes within the period gap. The largest orbital period a gPCEB can have that will evolve into the period gap within the lifetime of the Galaxy is given by the boundary $P_{\Re}^{+}$. Thus the total area from which gPCEBs can flow into the period gap within the Galactic lifetime is the region $A B C D$.

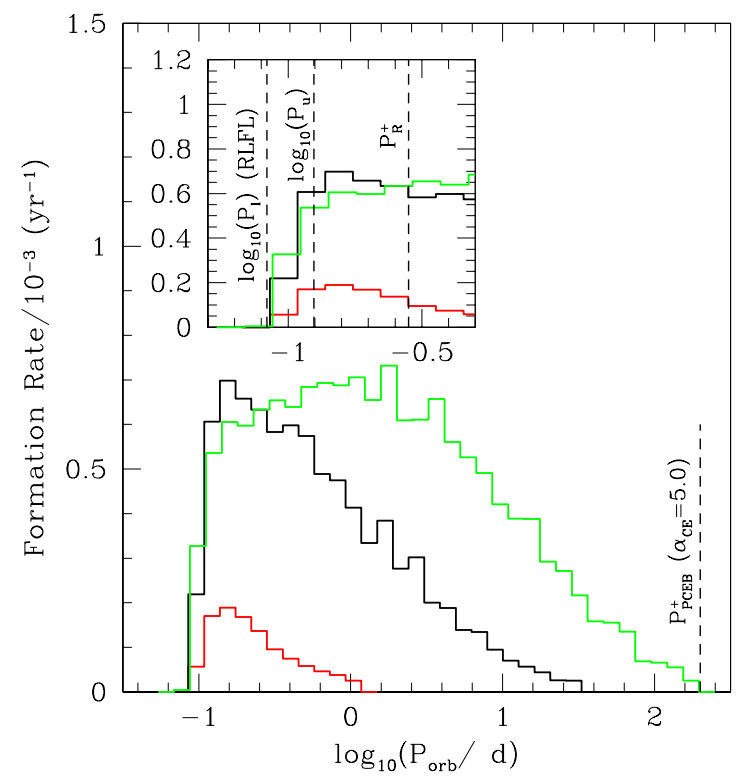

Figure 7. The formation rate of gPCEBs as a function of $\log _{10}\left(P_{\text {orb }} / \mathrm{d}\right)$ for $n(q)=1$ and for $\alpha_{\mathrm{CE}}=0.1$ (red), 1.0 (black) and 5.0 (green). Shown in the inset are the period gap boundaries $\log _{10} P_{\ell}$ (which is also the RLFL for $0.17 \mathrm{M} \odot$ secondaries) and $\log _{10} P_{\mathrm{u}}$, and $P_{\Re}^{+}$. The upper boundary $P_{\mathrm{PCEB}}^{+}$for $\alpha_{\mathrm{CE}}=5.0$ is shown in the main plot as the dashed line. Note that $P_{\mathrm{PCEB}}^{+}$, and in fact the whole distribution function, shifts to shorter periods as $\alpha_{\mathrm{CE}}$ decreases.

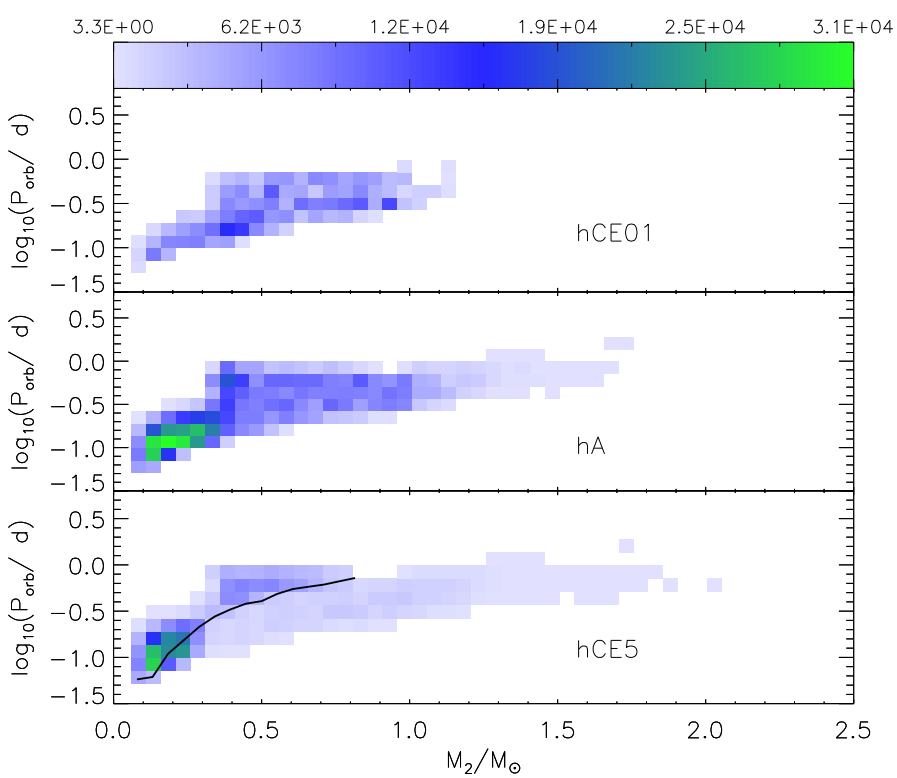

Figure 8. The formation rate of pre-CVs for $n(q)=1$ on the $M_{2}-\log _{10}\left(P_{\text {orb }}\right)$ plane for $\alpha_{\mathrm{CE}}=0.1$ (top panel), 1.0 (middle panel) and 5.0 (bottom panel). Pre-CVs that lie above the solid line in the bottom panel formed through a case $\mathrm{C} \mathrm{CE}$ phase, while those that lie below it formed through a case $\mathrm{B} \mathrm{CE}$ phase with a naked helium star remnant. The colour bar at the top indicates the formation rate $\left(\mathrm{Myr}^{-1}\right)$ per bin area $\mathrm{d} M_{2} \operatorname{dlog}_{10}\left(P_{\text {orb }}\right)$.

secondary mass is determined independently from the IMF according to (1). The largest population of gPCEBs and $\mathrm{dCVs}$ is obtained when the secondary mass is determined for this latter case. The smallest population numbers are found for $n(q) \propto q^{-0.99}$.

Combining the trends of $\mathrm{dCV}$ s and gPCEBs gives a decrease in the ratio of $\mathrm{dCV}$ :gPCEB with increasing $\alpha_{\mathrm{CE}}$, seen in the right-most panel of Figure 5 . For our reference IMRD function, dCV:gPCEB decreases from 12.9 for $\alpha_{\mathrm{CE}}=0.1$ to 0.9 for $\alpha_{\mathrm{CE}}=5.0$. Again this trend is the same for all forms of the IMRD function. We note that the ratio dCV:gPCEB decreases with decreasing value of $\nu$. To understand why, note that the $\mathrm{dCV}$ s will have formed from CVs filling their Roche lobes at long orbital periods above the period gap. This is in contrast to gPCEBs in the period gap which have secondary masses in the range $0.17 \leq M_{2} / M_{\odot} \leq 0.36$. Thus, for a given primary mass, the $\mathrm{dCV}$ progenitor systems will have, on average, more massive secondaries than found in gPCEBs. The IMRD $n(q) \propto q$ will mostly favour the formation of systems with more massive secondaries, thus mostly favouring the formation of $\mathrm{dCV}$ progenitors over the gPCEB progenitors. Thus we obtain the largest values of $\mathrm{dCV}: \mathrm{gPCEB}$. This is in contrast with the IMRD of the form $n(q) \propto q^{-0.99}$ which most favour the formation of systems with less massive secondaries, thereby mostly favouring the formation of gPCEB progenitors over dCV progenitors. We therefore obtain the smallest values of $\mathrm{dCV}$ :gPCEB out of the three forms of the IMRD. The smallest values of 
$\mathrm{dCV}$ :gPCEB are obtained when the secondary mass is determined independently from the primary mass.

\section{2 $\alpha_{\mathrm{CE}}$ as a function of Secondary Mass: models hPWR05 to hPWR2}

In models where $\alpha_{\mathrm{CE}}$ is determined from equation (6) both the present day populations of gPCEBs and dCVs decrease as the power index $p$ is increased. For our reference IMRD function, the left panel of Figure 9 shows that the number of gPCEBs decreases from $7.7 \times 10^{5}$ to $7.2 \times 10^{4}$. The middle panel shows that the number of dCVs decreases slightly from $4.1 \times 10^{6}$ to $3.1 \times 10^{6}$. As with the population of gPCEBs for models hA to hCE5, this trend depends on the flow rate of gPCEBs into the region $\Re$, and how this changes as the whole population of PCEBs is shifted with $\alpha_{\mathrm{CE}}$.

\subsubsection{The gPCEB Population}

The dark-grey area in Figure 2 indicates the range of secondary masses for the gPCEB systems. As $p$ increases from 0.5 to 2 , the average corresponding value of $\alpha_{\mathrm{CE}}$ for these systems decreases from $\sim 0.5$ to $\sim 0.1$. As a consequence the population of gPCEBs will shift to shorter orbital periods with increasing $p$. This in turn will change the formation rate of gPCEBs in the region $A B C D$ and hence the flux $F_{\Re}$.

As can be seen in the left panel of Figure 5 a value of $\alpha_{\mathrm{CE}}$ decreasing from $\sim 0.5$ to $\sim 0.1$ leads to the decrease in the present-day number of gPCEBs in the period gap. This corresponds to the decrease in the present-day number of gPCEBs with increasing $p$ seen in the left panel of Figure 9

\subsubsection{The $d C V$ Population}

As with the dCVs for models hA to hCE5, the population of dCVs for hPL05 to hPL2 depends on the formation rate of pre-CVs with $M_{2}>M_{\mathrm{MS} \text {, conv }}$ above the period gap. The light-grey region in Figure 2 shows the range of secondary masses for $\mathrm{dCV}$ progenitor systems. For this range in secondary mass there is, on average, little change in $\alpha_{\mathrm{CE}}$. Indeed, the dominant secondary mass group for pre-CV progenitors is $M_{2} \lesssim 0.6 \mathrm{M}_{\odot}$; here an increase in $p$ from 0.5 to 2 corresponds to a decrease in $\alpha_{\mathrm{CE}}$ from $\sim 0.7$ to $\sim 0.3$. Hence from the middle panel of Figure [5] a slight decrease in $N_{\mathrm{dCV}}$ can be expected. This is indeed the case as shown in the middle panel of Figure 9

\subsubsection{Overall Trends}

The trend in the present day populations of gPCEBs and $\mathrm{dCVs}$ with $p$ is the same for all forms of the IMRD function, and where the secondary mass is determined from the same IMF as the primary mass. As before, the largest population of dCVs and gPCEBs occurs when the secondary mass is determined from the same IMF as the primary. The smallest populations occur when $n(q) \propto q^{-0.99}$.

As a consequence of the behaviour discussed in Sections 3.2.1 and 3.2.2, the ratio dCV:gPCEB increases as $p$ increases. For our reference model, dCV:gPCEB increases from 5.3 for $p=0.5$ to 43.1 for $p=2$. The variation of
$\mathrm{dCV}$ :gPCEB with $p$ is shown in the most right hand panel in Figure 9

Note that these ratios are very large for $p=2$ as PCEBs with low-mass donors will encounter a merger. Hence the vast majority of all CVs will form above the period gap, with very few gPCEBs within the period gap. Politano \& Weiler (2007) suggested that such a strong dependence of $\alpha_{\mathrm{CE}}$ on $M_{2}$ could explain the lack of CVs with sub-stellar secondaries. However, we note that a lack of calculated gPCEBs with low-mass secondaries appears to be inconsistent with observations (Ritter \& Kolb 2003, catalogue Edition 7.7 (2006)), which show a modest number of PCEBs with $M_{2} \approx 0.17 \mathrm{M}_{\odot}$. We therefore believe that the $p=2$ model is not a realistic one. As with models hA to hCE5, $\mathrm{dCV}$ :gPCEB decreases with decreasing values of $\nu$. The reasons for this are the same as those described in Section 3.1. The smallest value of $\mathrm{dCV}$ :gPCEB occurs when the secondary mass is determined independently from the primary mass.

\subsection{Alternative Magnetic Braking Laws: Models rvj2A and rvj4A}

We now consider the impact of different forms of magnetic braking on gPCEBs and dCVs. Increasing $\gamma$ from 2 to 4 increases the present day number of $\mathrm{dCVs}$ from $5.5 \times 10^{6}$ for $\gamma=2$ to $6.0 \times 10^{6}$ for $\gamma=4$, for our reference IMRD. The Rappaport et al. (1983) prescription of magnetic braking gives a larger present day population of dCVs than the Hurley et al. (2002) prescription, between any IMRD function; for our reference model hA, with $n(q)=1$, the present day number of $\mathrm{dCVs}$ is $3.5 \times 10^{6}$.

The pre-CV progenitor systems of $\mathrm{CVs}$ that form above the period gap will initially be driven by magnetic braking. Increasing the strength of magnetic braking will shift the upper limit $P_{\text {preCV }}^{+}$to longer orbital periods for systems with $M_{2}>M_{\mathrm{MS} \text {,conv }}$. Thus more systems will become semidetached within the lifetime of the Galaxy. From equations (25) to (26), this will result in an increase in the formation rate of pre-CVs above the period gap, and therefore increase the formation rate of dCVs. In turn, this will increase the present day population of dCVs.

Note that the number of present day gPCEBs does not change with magnetic braking law. In our model, the mass at which isolated stars become fully convective is $0.35 \mathrm{M}_{\odot}$ (Hurley et al. 2002). Thus, the evolution of gPCEBs with $M_{2} \leq 0.35 \mathrm{M}_{\odot}$ will be driven by gravitational radiation only. As we are only considering PCEBs with secondaries in the range $0.17 \leq M_{2} / M_{\odot} \leq 0.36$, only very few systems will evolve via magnetic braking while the larger majority will be driven via gravitational radiation.

These trends in the population of $\mathrm{dCV}$ s and gPCEBs result in an increase in $\mathrm{dCV}$ :gPCEB with increasing magnetic braking strength. For our reference IMRD function, and where $\alpha_{\mathrm{CE}}=1.0$, the ratio increases from 3.5 for the Hurley et al. (2002) form of magnetic braking, to 6.0 for the Rappaport et al. (1983) prescription, $\gamma=4$. The largest values of $\mathrm{dCV}$ :gPCEB occur when the IMRD function is $n(q) \propto q$, while the smallest occur when $n(q) \propto q^{-0.99}$. 

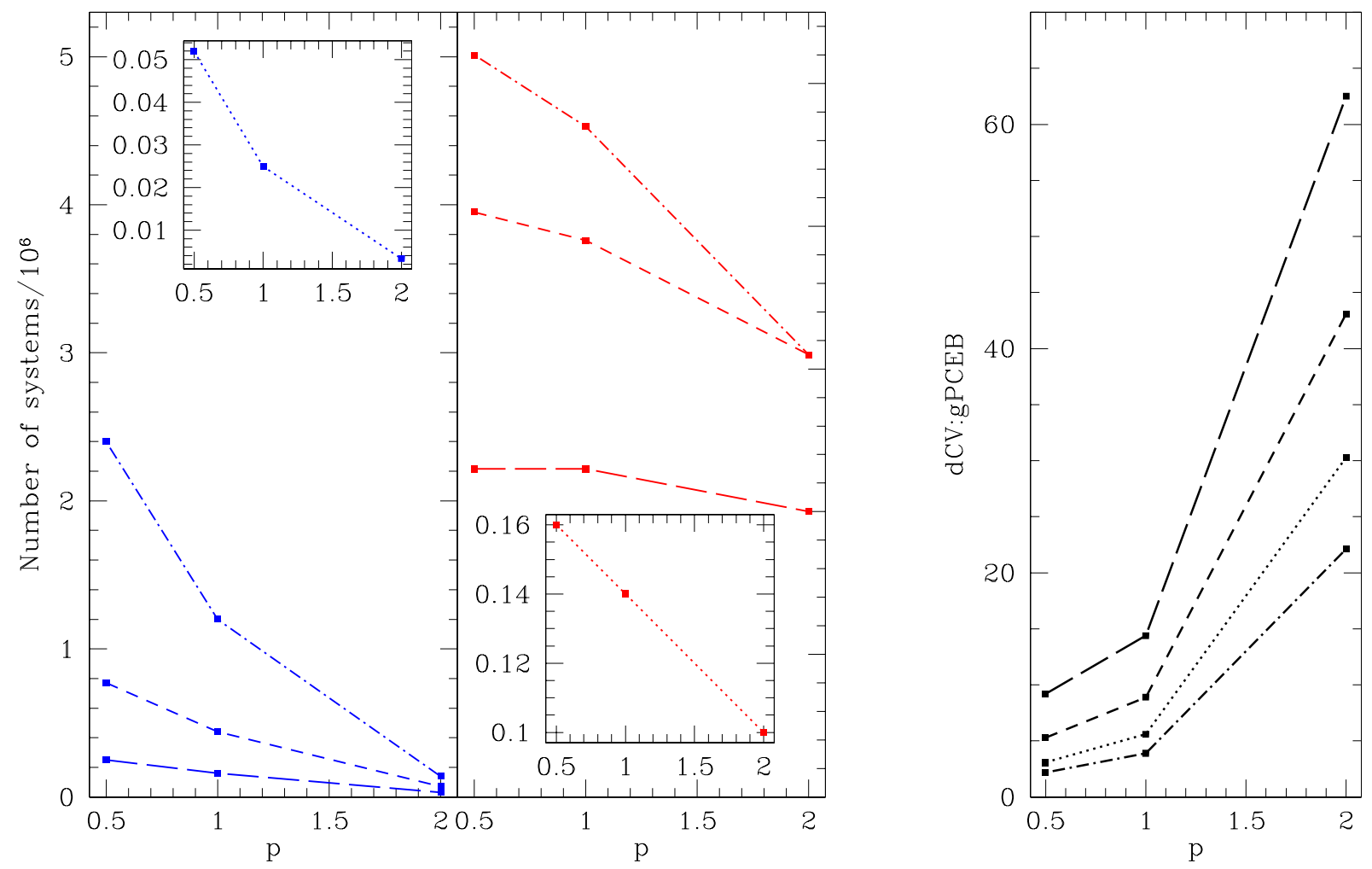

Figure 9. From left to right: the present day population of gPCEBs in the period gap (blue) and dCVs (red) as a function of the power index $p$, and the ratio $\mathrm{dCV}$ :gPCEB as a function of $p$. Long-dashed: $n(q) \propto q$; short-dashed: $n(q)=1$; dot-dashed: where $M_{2}$ is picked independently from the same IMF as $M_{1}$ according to equation (1); dotted: $n(q) \propto q^{-0.99}$ (insets).

\section{DISCUSSION}

\subsection{Observational Predictions}

In this section we consider the number of present day dCVs and gPCEBs, and hence the ratio dCV:gPCEB, given the commonly assumed form of the IMRD and values of $\alpha_{\mathrm{CE}}$. We can then gauge whether such an excess of dCVs over gPCEBs can be determined observationally.

Numerical calculations of the CE phase suggest that $\alpha_{\mathrm{CE}}=0.13$ to 0.6 (Iben \& Livio 1993), while the IMRD seems to be flat in $q$, i.e. $n(q)=1$ (Goldberg, Mazeh \& Latham 2003; Mazeh et al. 1992; Duquennoy \& Mayor 1991). From Table 2 we can estimate that the number of dCVs currently occupying the Galaxy is $(2.2-3.8) \times 10^{6}$, while the number of gPCEBs is in the range $(1.7-8.6) \times 10^{5}$, giving a factor of $\sim 4$ to $\sim 13$ more dCVs than gPCEBs. Hence we predict a prominent peak in the orbital period distribution of short orbital period WDMS systems as shown in Figure 10 In determining these predictions, we have assumed that magnetic braking takes the form shown in equation (8).

Of course, the AM loss rate associated with magnetic braking is not very well constrained, and indeed it has been suggested that the magnetic braking strength is $\approx 100$ weaker than the forms we have used in this investigation (Andronov et al. 2003). However, we have not considered the forms of magnetic braking that include a saturation point if the secondary star has a spin frequency larger than some critical value $\Omega_{\text {crit }}$ (Sills et al. 2000; Ivanova \& Taam 2003).This is because, in the case of the Sills et al. (2000) form of magnetic braking, the AM loss rate would be too small to sufficiently drive the donor star out of thermal equilibrium, and consequently produce a period gap. Furthermore, the Sills et al. (2000) form of magnetic braking predict mass transfer rates at the upper edge of the period gap that are too low compared to observations, and as such would not explain the presence of nova-like CVs above the period gap (e.g. Kolb 2002). The Ivanova \& Taam (2003) form of magnetic braking on the other hand, which is $\sim 2$ to 3 times weaker than the Verbunt \& Zwaan (1982) form, would drive the donor star out of thermal equilibrium but not enough to explain the observed width of the period gap.

Such forms of magnetic braking are therefore inconsistent with the disrupted magnetic braking model. Instead, alternative mechanisms must exist that combine in strength to reach the value that we consider at the upper edge of the period gap.

\subsection{The Location of the Period Gap}

We now discuss the impact the location of the period gap has on our results. We note that Knigge (2006), using a similar method to ours, determined that the period gap edges are $2.15 \mathrm{~h}$ and $3.18 \mathrm{~h}$, which agrees with our determined period gap width of one hour. We attribute the slight differ- 


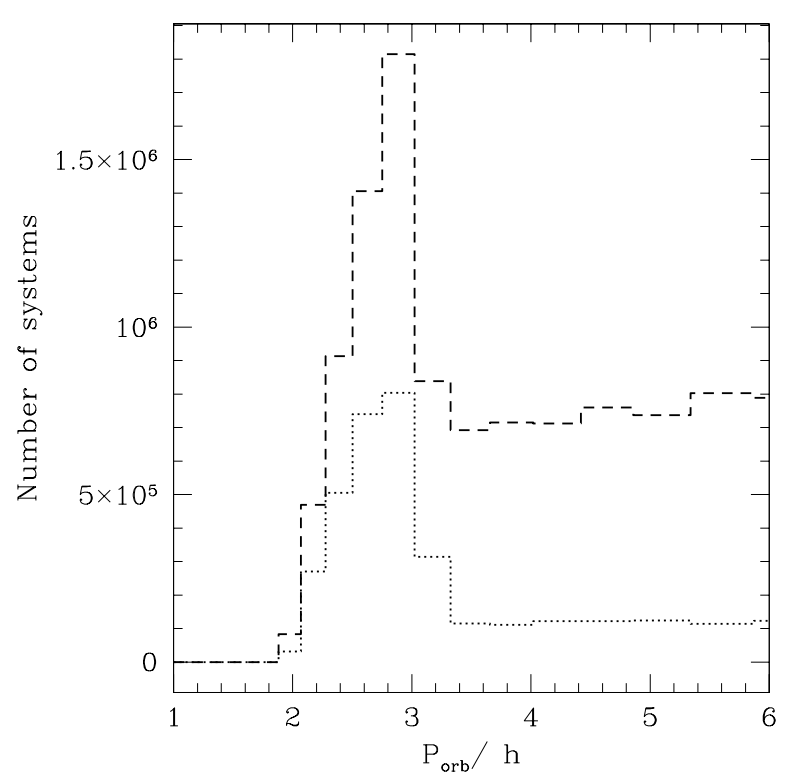

Figure 10. The orbital period distribution of the combined population of dCVs and gPCEBs for $n(q)=1, \alpha_{\mathrm{CE}}=0.6$ (dashed line) and $\alpha_{\mathrm{CE}}=0.1$ (dotted line).

ence between the two locations of the period gap to the two different catalogues used. Knigge (2006) used the Ritter \& Kolb (2003) catalogue (Edition 7.6), while we use the latest Edition 7.7.

If the period gap was located according to Knigge (2006), this would have the effect of increasing the population of gPCEBs within the period gap, and consequently decrease the ratio dCV:gPCEB. The higher values of $P_{\mathrm{u}}$ and $P_{\ell}$ would shift the mass range of gPCEBs to $0.19 \leq$ $M_{2} / \mathrm{M}_{\odot} \leq 0.40$, and therefore a larger fraction of gPCEBs (those with $0.35<M_{2} / \mathrm{M}_{\odot} \leq 0.40$ ) would be driven by magnetic braking. In turn, the flow rate of gPCEBs from longer orbital periods into $\Re$ would increase. We also point out that a similar uncertainty is introduced with the value of $M_{\mathrm{MS}, \mathrm{conv}}$, as this is not well constrained by stellar evolution models.

In their studies on circumbinary disk driven CVs Willems et al. (2005) considered the CV population below the gap, while Willems et al. (2007) studied the population above the gap. These authors never attempted to systematically model, or to achieve a best fit model for, the combined population. This is why their adopted period gap boundaries of 2.25 and 2.75 hours, respectively, result in an implausibly narrow gap when taken together.

Nonetheless we investigated how a narrower period gap would affect our results by calculating a further population model with the gap boundaries set to 2.25 and 2.75 hours. We considered $\alpha_{\mathrm{CE}}=1.0$ with our standard IMRD, and recalibrated equation (8) to give the AM loss rate appropriate for the narrower gap width. We find that dCV:gPCEB decreases to 2.1 compared to our model hA value of 3.5. Thus the prominence of the peak would decrease somewhat. This is because the time for a dCV to cross a narrower gap would decrease (we find $\left\langle\tau_{\mathrm{dCV}}\right\rangle \approx 438 \mathrm{Myr}$ ), decreasing the present day population from $3.5 \times 10^{6}$ for model hA to $1.1 \times 10^{6}$. The number of gPCEBs in the period gap also decreases from $1.0 \times 10^{6}$ for model hA to $5.2 \times 10^{5}$ because the systems occupy a smaller area in period space.

We emphasise that a gap width of half an hour is a somewhat extreme assumption. Despite this we still obtain a clear excess of dCVs over gPCEBs in the period gap, and hence a prominent peak would still be detected. Indeed, a prominent peak would still be detected for any reasonable gap width.

\subsection{TTMT Systems}

Observational investigations have shown that in many CVs above the period gap the donor is unlikely to be an unevolved main sequence donor (e.g. Beuermann et al. 1998). Instead, the spectral types of the donor are later than those of an isolated star of the same mass, while donors at orbital periods longer than 5 to 6 hours are nuclear evolved (Patterson et al. 2005; Knigge 2006). Theoretical calculations also predict this effect (Baraffe \& Kolb 2000). It has been suggested that such systems had an initial TTMT phase where the secondary star was much more massive than the white dwarf (Schenker \& King 2002). Kolb \& Willems (2005) find that as much as 40 per cent of zero-age CVs form with a donor that has evolved more than half way through its main sequence lifetime. Gänsicke et al. (2003) have detected CNO abundance anomalies consistent with nuclear evolved donors among $\sim 10-15$ per cent CVs in a sample observed in the ultraviolet, corroborating the hypothesis that a significant fraction of the known CV population may be post-TTMT systems.

Such CVs, if they appear after their initial TTMT phase above the period gap, may contribute to the present day population of dCVs. Thus our values of dCV:gPCEB, which are calculated from the evolution of purely AM driven CVs, are lower limits. To calculate the contribution of post-TTMT $\mathrm{CVs}$ to the total dCV population would require a comprehensive treatment of the TTMT phase including the fate of the transferred material, which determines the point of re-appearance of the system as a 'normal' AM driven CV.

\subsection{Magnetic CVs}

We now consider how the contribution of magnetic CVs (mCVs) may affect the ratio dCV:gPCEB within the period gap. Intermediate polars (IPs) are thought to evolve as non-magnetic $\mathrm{CVs}$ and hence become detached at the upper edge of the period gap due to the disruption of magnetic braking. Their contribution to the population of dCVs is therefore already taken into account in our calculations, as are their progenitors to the gPCEB population.

Polars, on the other hand, may not become detached at the upper edge of the period gap, as it has been suggested that magnetic braking for these systems is estimated to be 1 to 2 orders of magnitude weaker than for nonmagnetic CVs (Li, Wu \& Wickramasinghe 1994; Li, Wu \& Wickramasinghe 1994b). This is supported observationally from their white dwarf temperatures: the accretion-heated white dwarfs in polars are consistently colder than those in non-magnetic CVs at similar orbital periods, implying lower accretion rates, and hence angular momentum loss rates (Araujo-Betancor et al. 2005). This difference is es- 
pecially pronounced above the period gap, where magnetic braking is the dominant AM loss agent.

Furthermore, no progenitor WDMS systems of mCVs with a magnetic white dwarf has as yet been detected (Liebert et al. 2005). It is possible that such progenitors evolve in the same way as progenitors of non-magnetic CVs. In such a case, the population of WDMS progenitors of polars will already be taken into account in our calculations and are included in the number of gPCEBs we obtained.

The overall effect of the above on our results would be to reduce the number of $\mathrm{dCVs}$ and hence the ratio $\mathrm{dCV}$ :gPCEB by the fraction of polars among the total CV population. From the Ritter \& Kolb (2002) catalogue, Edition 7.7 (2006), we find that polars contribute $\sim 13$ per cent to the total $\mathrm{CV}$ population.

\subsection{PCEB Candidates}

Here we discuss the current situation regarding PCEB candidates and the surveys presently searching for them. Schreiber \& Gänsicke (2003) studied 30 well observed PCEBs, which were chosen to be representative of PCEBs that will begin mass transfer within the Hubble time. These systems have orbital periods $\lesssim 2$ days, have mass ratios less than 1, and do not include secondaries that are sub-giants. They found that the majority of systems contained young $\left(\lesssim 5 \times 10^{8} \mathrm{yrs}\right)$, hot $(\approx 15000-22000 \mathrm{~K})$ white dwarfs. This is a consequence of the fact that until recently PCEB candidates were almost exclusively identified in blue colour surveys such as the Palomar Green survey. Thus, systems containing cool white dwarfs and/or early type companion stars have been missed in most previous work, with the exception of a few systems identified as nearby large proper motion systems (e.g. RR Cae) or spectroscopic binaries (e.g. V471 Tau).

The potential of observational population studies of PCEBs has improved over the past few years dramatically through the SDSS. Because of the vast ugriz colour space probed by SDSS, coupled with the availability of highquality follow-up spectroscopy of a large number of objects with non-stellar colours, has already lead to the identification of more than 1000 WDMS binaries (Silvestri et al. 2007), most of them being by-products of the search for quasars. A complementary program targeting specifically WDMS binaries containing cool white dwarfs and/or early type companions is underway to compensate the bias against such systems in the previous surveys (Schreiber et al. 2007). For the first phase, the PCEBs among the full sample of WDMS binaries have to be identified through radial velocity studies. A first effort along these lines has been carried out by Rebassa-Mansergas et al. (2007) who identified 18 PCEB candidates from multiple SDSS spectroscopy obtained for 101 WDMS. Rebassa-Mansergas (2008, in preparation) confirmed through follow-up spectroscopy so far six of those candidates as PCEBs with orbital periods ranging from $164 \mathrm{~min}$ to $1048 \mathrm{~min}$. Additional identification work of the PCEBs among the SDSS WDMS is currently carried out at the VLT (Schreiber et al. 2008) and the WHT (Gänsicke et al. in preparation).

It is foreseeable that, sufficient observational effort being invested, it will be possible to build up an orbital period distribution of close WDMS binaries comprising potentially a few hundred systems. While the SDSS PCEB sample will not be free of selection effects (see e.g. Rebassa-Mansergas et al. 2007; Pretorius, Knigge \& Kolb 2006), those biases can be modelled to a large degree. It appears hence feasible to subject the disrupted magnetic braking model to a stringent test by comparing such an observed orbital period distribution to the predictions of our work here (Figure 10).

\section{CONCLUSIONS}

We have performed population synthesis calculations to obtain the present day population of two types of white dwarfmain sequence star systems within the 2 to 3 hour cataclysmic variable period gap. The first are post-CE binaries with secondaries that have masses $0.17 \leq M_{2} / \mathrm{M}_{\odot} \leq 0.36$, and so will commence mass transfer in the period gap (gPCEBs). The second type are systems that were CVs in the past, but detached at the upper edge of the period gap as a consequence of disrupted magnetic braking, and are crossing the period gap via gravitational radiation ( $\mathrm{dCVs}$ ).

Our calculations were repeated to consider constant, global values of the $\mathrm{CE}$ ejection efficiency, $\alpha_{\mathrm{CE}}$, and cases where $\alpha_{\mathrm{CE}}$ is a function of secondary mass according to equation (6). We considered various forms of magnetic braking according to equation (7) with $\gamma=2$ and 4 , and equation (8).

We find that there is a prominent peak in the orbital period distribution of the combined $\mathrm{dCV}$ and $\mathrm{gPCEB}$ population, due to the excess of dCVs over gPCEBs within the period gap. We find that the ratio $\mathrm{dCV}$ :gPCEB, which gives an indication of the peak's height, decreases with an increasing global value of $\alpha_{\mathrm{CE}}$, while increasing with increasing value of $p$. These trends are the same for all assumptions on the initial secondary mass distribution. The value of $\mathrm{dCV}$ :gPCEB ranges from 0.5 for model hCE5 where the secondary mass is determined independently from the primary mass using the same IMF, to 62.5 for model hPWR2 where $n(q) \propto q$.

We find further that $\mathrm{dCV}: \mathrm{gPCEB}$ increases with increasing magnetic braking strength, although only slightly. For $n(q)=1, \mathrm{dCV}$ :gPCEB increases from 3.5 for model hA (the weakest) to 6.0 for $\mathrm{rvj} 4 \mathrm{~A}$ (the strongest).

For all our models we find that the values of $\mathrm{dCV}$ :gPCEB are largest when the IMRD function has the form $n(q) \propto q$ i.e. on average systems will have more massive secondary stars. The smallest values occur when the secondary mass is determined from the same IMF as the primary.

The most likely value of $\mathrm{dCV}: \mathrm{gPCEB}$ is between $\sim 4$ to $\sim 13$, thus we can expect a significant peak as shown in Figure 10. We suggest that if such a feature is observed in the orbital period distribution of short orbital period WDMS binaries, this would strongly corroborate the disruption of magnetic braking.

\section{ACKNOWLEDGEMENTS}

PJD acknowledges studentship support from the Science \& Technology Facilities Council. BW acknowledges partial support from NASA BEFS grant NNG06GH87G and BSF 
CAREER Award AST-0449558 to Vicky Kalogera at Northwestern University. We thank the anonymous referee for comments that helped to improve the presentation of the paper.

\section{REFERENCES}

Andronov N., Pinsonneault M., Sills A., 2003, ApJ, 582, 358

Araujo-Betancor, S., Gänsicke, B. T., Long, K. S., Beuermann, K., de Martino, D., Sion, E. M., Szkody, P., 2005, ApJ, 622, 589

Baraffe I., Kolb U., 2000, MNRAS, 318, 354

Beuermann K., Baraffe I., Kolb U., Weichhold M., 1998, A\&A, 339, 518

Collier-Cameron A., 2002, in Gänsicke B. T., Beuermann K., Reinsch K., eds, ASP Conf. Ser., vol. 261, The Physics of Cataclysmic Variables and Related Objects, p. 11

Duquennoy A., Mayor M., 1991, A\&A, 248, 485

Gänsicke et al., 2003, ApJ, 594, 443

Goldberg D., Mazeh T., Latham D. W., 2003, ApJ, 591, 397

Hurley J. R., Pols O. R., Tout C. A., 2000, MNRAS, 315, 543

Hurley J. R., Pols O. R., Tout C. A., 2002, MNRAS, 329, 897

Iben I., Tutukov A. V., 1984, ApJS, 54, 235

Iben I. J., Livio M., 1993, PASP, 105, 1357

Ivanova N., Taam R. E., 2003, ApJ, 599, 516

Jones B. F., Fischer D. A., Stauffer J. R., 1996, AJ, 112, 1562

Kalogera V., Henniger M., Ivanova N., King A. R., ApJ, 603, L41

King A. R., 1988, QJRAS, 29, 1

King A. R., Kolb U., 1995, ApJ, 439, 330

Knigge C., 2006, MNRAS, 373, 484

Kolb U., 1996, in Evans A., Wood J. H., eds, Astrophysics and Space Sci. Lib.,IAU Colloq. 158: Cataclysmic Variables and Related Objects, Vol. 208, p. 433

Kolb U., Stehle R., 1996, MNRAS, 282, 1454

Kolb U., King A. R., Ritter H., 1998, MNRAS, 298, L29

Kolb U., 2002, in Gänsicke B. T., Beuermann K., Reinsch K., eds, ASP Conf. Ser. Vol. 261, The Physics of Cataclysmic Variables and Related Objects, p. 180

Kolb U., Willems B., 2005, in Hameury J. M., Lasota J. P., eds, ASP Conf. Ser. Vol. 330, Thermal-timescale mass transfer and evolved donor stars in CVs, p. 17

Kroupa P., Tout C. A., Gilmore G., 1993, MNRAS, 262, 545

Li J. K., Wu K. W., Wickramasinghe D. T., 1994, MNRAS, 268,61

Li J. K., Wu K. W., Wickramasinghe D. T., 1994b, MNRAS, 270, 769

Liebert J., Wickramsinghe D. T., Schmidt G. D., Silvestri N. M., Hawley S. L., Szkody P., Ferrario L., Webbink R. F., Oswalt T. D., Smith J. A., Lemagie M. P., 2005, ApJ, 129, 2376

Mazeh T., Goldberg D., Duquennoy A., Mayor M., 1992, ApJ, 401, 265

McDermott P. N., Taam R. E., 1989, ApJ, 342, 1019

Paczyński B., 1971, ARA\&A, 9, 183
Paczyński P., 1976, in Eggleton P., Mitton S., Whelan J., eds, Proc. IAU Symp. 73, Structure and Evolution of Close Binary Systems, p. 75

Patterson J., et al., 2005, PASP, 117, 1204

Politano M., Weiler K. P., 2006, ApJ, 641, L137

Politano M., Weiler K. P., 2007, ApJ, 665, 663

Rappaport S., Verbunt F., Joss P. C., 1983, ApJ, 275, 713

Pretorius M. L., Knigge C., Kolb U., 2007, MNRAS, 374, 1495

Rebassa-Mansergas M. R., Gänsicke B. T., Schreiber M. R., Koester D., 2007, MNRAS, 382, 1337

Ritter H., 1988, A\&A, 202, 93

Ritter H., Kolb U., 2003, A\&A, 404, 301; edition RKcat 7.7 (2006)

Schenker K., 2001, in Podsiadlowski Ph., Rappaport S., King A. R., D'Antona F., Burderi L., eds, ASP Conf. Ser. Vol. 229, Evolution of Binary and Multiple Star Systems, p. 321

Schenker K., King A. R., 2002, in Gänsicke B. T., Beuermann K., Reinsch K., eds, ASP Conf. Ser., vol. 261, The Physics of Cataclysmic Variables and Related Objects, p. 242

Schreiber M. R., Gänsicke B. T., 2003, A\&A, 406, 305

Schreiber M. R., Nebot Gomez-Moran A., Schwope A., 2007, in Napiwotzki R., Burleigh R., eds. 15th. European Workshop on White Dwarfs, ASP Conf. Ser., 372, 459

Schreiber, M. R., Gaensicke, B. T., Southworth, J., Schwope, A. D., Koester, D., 2008, A\&A, in press, arXiv:0709.4545

Silvestri, N. M., et al., 2007, AJ, 134, 741

Skumanich A., 1972, ApJ, 171, 565

Sills A., Pinsonneault M. H., Terndrup D. M., 2000, ApJ, 534,335

Smith D. A., Dhillon V. S., 1998, MNRAS, 301, 767

Spruit H. C., Ritter H., 1983, A\&A, 124, 267

Stehle R., Ritter H., Kolb U., 1996, MNRAS, 279, 581

Taam R. E., Spruit H. C., 1989, ApJ, 345, 972

Townsley, D.M., Bildsten, L., 2003, ApJ, 596, L227

Verbunt F., Zwaan C., 1981, A\&A, 100, L7

Warner B., 1995, Cataclysmic Variable Stars, Cambridge Univ. Press, Cambridge

Weidemann V., 1990, ARA\&A, 28, 103

Willems B., Kolb U., 2002, MNRAS, 337, 1004

Willems B., Kolb U., 2004, A\&A, 419, 1057

Willems B., Kolb U., Sandquist E. L., Taam R. E., Dubus G., 2005, ApJ, 635, 1263

Willems B., Taam R. E., Kolb U., Dubus G., Sandquist E. L., 2007, ApJ, 657, 465 